Supporting Information

for

\title{
Calculations Predict that Heavy-Atom Tunneling Dominates Möbius Bond Shifting in [12]- and [16]Annulene
}

Jessica K. Arbitman, Cameron S. Michel, Claire Castro, and William L. Karney

Page Contents

S-2 Table S1. M06-2X/cc-pVDZ Absolute Energies and ZPEs for $\mathrm{C}_{12} \mathrm{H}_{12}$ Stationary Points

S-2 Table S2. CCSD(T)/cc-pVDZ//M06-2X/cc-pVDZ Absolute Energies for $\mathrm{C}_{12} \mathrm{H}_{12}$ Stationary Points

S-3 Table S3. CCSD(T)/cc-pVTZ//M06-2X/cc-pVDZ Absolute Energies for $\mathrm{C}_{12} \mathrm{H}_{12}$ Stationary Points

S-3 Table S4. M06-2X/cc-pVDZ Absolute Energies and ZPEs for $\mathrm{C}_{16} \mathrm{H}_{16}$ Stationary Points

S-4 Table S5. CCSD(T)/cc-pVDZ//M06-2X/cc-pVDZ Absolute Energies for $\mathrm{C}_{16} \mathrm{H}_{16}$ Stationary Points

S-4 Note on Methods for Computing Tunneling Rate Constants

S-5 Figure S1. Arrhenius plot for 6-electron electrocyclic ring closure of CCCTCT[12]annulene

S-6 Figure S2. M06-2X/cc-pVDZ optimized geometries of $\mathrm{C}_{12} \mathrm{H}_{12}$ stationary points

S-7 Figure S3. M06-2X/cc-pVDZ optimized geometries of $\mathrm{C}_{16} \mathrm{H}_{16}$ stationary points

S-8 Figure S4. Plots of energy (Vmep) and C-C bond lengths as a function of massscaled reaction coordinate, for bond shifting steps in [12]- and [16]annulene

S-9 Sample Input Files for Polyrate Calculations

S-14 Table S6. Computed Rate Constants for Möbius Bond Shifting in CTCTCT[12]Annulene

S-15 Table S7. Computed Rate Constants for Electrocyclization of [12]annulene

S-16 Table S8. Computed Rate Constants for Möbius Bond Shift in [16]Annulene

S-17 M06-2X/cc-pVDZ Optimized Cartesian Coordinates for Stationary Points

S-21 Complete Citations for Gaussian and Polyrate 
Table S1. M06-2X/cc-pVDZ Absolute Energies and ZPEs for $\mathrm{C}_{12} \mathrm{H}_{12}$ Stationary Points ${ }^{\mathrm{a}}$

\begin{tabular}{lcccccc}
\hline $\mathrm{cpd}$ & $\mathrm{sym}$ & $\begin{array}{c}\mathrm{E} \\
(\mathrm{au})\end{array}$ & $\begin{array}{c}\text { NIMAG } \\
\left(\omega, \mathrm{cm}^{-1}\right)\end{array}$ & $\begin{array}{c}\text { ZPE } \\
(\mathrm{kcal} / \mathrm{mol})\end{array}$ & $\begin{array}{c}\text { rel E, uncorr } \\
(\mathrm{kcal} / \mathrm{mol})\end{array}$ & $\begin{array}{c}\text { rel E }(0 \mathrm{~K}) \\
(\mathrm{kcal} / \mathrm{mol})\end{array}$ \\
\hline $\mathbf{1}$ & $C_{1}$ & -464.21354 & 0 & 126.46 & 0.0 & 0.0 \\
$\mathbf{T S}(\mathbf{1} \rightarrow \mathbf{2 b})$ & $C_{1}$ & -464.18405 & $1(718 i)$ & 125.37 & 18.5 & 17.4 \\
$\mathbf{2 b}$ & $C_{1}$ & -464.20014 & 0 & 125.96 & 8.4 & 7.9 \\
$\mathbf{T S}(\mathbf{2 b} \rightarrow \mathbf{2 a})$ & $C_{1}$ & -464.19831 & $1(101 i)$ & 125.45 & 9.6 & 8.6 \\
$\mathbf{2 a}$ & $C_{\mathrm{S}}$ & -464.20682 & 0 & 126.07 & 4.2 & 3.8 \\
$\mathbf{T S}(\mathbf{2 a} \rightarrow \mathbf{3})$ & $C_{\mathrm{S}}$ & -464.18567 & $1(476 i)$ & 125.89 & 17.5 & 16.9 \\
$\mathbf{3}$ & $C_{\mathrm{S}}$ & -464.23092 & $1(66 i)$ & 127.61 & -10.9 & -9.8 \\
\hline
\end{tabular}

${ }^{\text {a }}$ All relative energies are given relative to 1 . "rel $\mathrm{E}(0 \mathrm{~K})$ " includes correction for differences in ZPE. Bicyclic compound $\mathbf{3}$ has $C_{1}$ symmetry, but the intrinsic reaction coordinate (IRC) and direct dynamics calculations from $C_{\mathrm{S}}$ symmetric $\operatorname{TS}(\mathbf{2 a} \rightarrow \mathbf{3})$ lead down the $C_{\mathrm{s}}$ symmetric ridge, so the energy of the $C_{\mathrm{s}}$ species is given. This does not affect the rate calculations due to the significant exothermicity of the reaction $\mathbf{2 a} \rightarrow \mathbf{3}$.

Table S2. CCSD(T)/cc-pVDZ//M06-2X/cc-pVDZ Absolute Energies for $\mathrm{C}_{12} \mathrm{H}_{12}$ Stationary

Points $^{\mathrm{a}}$

\begin{tabular}{lccccc}
\hline cpd & sym & $\begin{array}{c}\mathrm{CCSD}(\mathrm{T}) \\
\mathrm{E}(\mathrm{au})\end{array}$ & $\begin{array}{c}\mathrm{T}_{1} \\
\text { diagnostic }\end{array}$ & $\begin{array}{c}\text { rel E, uncorr } \\
(\mathrm{kcal} / \mathrm{mol})\end{array}$ & $\begin{array}{c}\text { rel E }(0 \mathrm{~K}) \\
(\mathrm{kcal} / \mathrm{mol})\end{array}$ \\
\hline $\mathbf{1}$ & $C_{1}$ & -463.05914 & 0.012 & 0.0 & 0.0 \\
$\mathbf{T S}(\mathbf{1} \rightarrow \mathbf{2 b})$ & $C_{1}$ & -463.02828 & 0.015 & 19.4 & 18.3 \\
$\mathbf{2 b}$ & $C_{1}$ & -463.04666 & 0.012 & 7.8 & 7.3 \\
$\mathbf{T S}(\mathbf{2 b} \rightarrow \mathbf{2 a})$ & $C_{1}$ & -463.04539 & 0.011 & 8.6 & 7.6 \\
$\mathbf{2 a}$ & $C_{\mathrm{S}}$ & -463.05264 & 0.011 & 4.1 & 3.7 \\
$\mathbf{T S}(\mathbf{2 a} \rightarrow \mathbf{3})$ & $C_{\mathrm{S}}$ & -463.02967 & 0.011 & 18.5 & 18.0 \\
$\mathbf{3}$ & $C_{\mathrm{S}}$ & -463.07638 & 0.011 & -10.8 & -9.7 \\
\hline
\end{tabular}

${ }^{a}$ All relative energies are given relative to 1 . "rel E $(0 \mathrm{~K})$ " includes correction for differences in ZPE. 
Table S3. CCSD(T)/cc-pVTZ //M06-2X/cc-pVDZ Absolute Energies for $\mathrm{C}_{12} \mathrm{H}_{12}$ Stationary Points $^{\mathrm{a}}$

\begin{tabular}{lccccc}
\hline cpd & sym & $\begin{array}{c}\mathrm{CCSD}(\mathrm{T}) \\
\mathrm{E}(\mathrm{au})\end{array}$ & $\begin{array}{c}\mathrm{T}_{1} \\
\text { diagnostic }\end{array}$ & $\begin{array}{c}\text { rel E, uncorr } \\
(\mathrm{kcal} / \mathrm{mol})\end{array}$ & $\begin{array}{c}\text { rel E }(0 \mathrm{~K}) \\
(\mathrm{kcal} / \mathrm{mol})\end{array}$ \\
\hline $\mathbf{1}$ & $C_{1}$ & -463.50653 & 0.012 & 0.0 & 0.0 \\
$\mathbf{T S}(\mathbf{1} \rightarrow \mathbf{2 b})$ & $C_{1}$ & -463.47598 & 0.015 & 19.2 & 18.1 \\
$\mathbf{2 b}$ & $C_{1}$ & -463.49432 & 0.012 & 7.7 & 7.2 \\
$\mathbf{T S}(\mathbf{2 b} \rightarrow \mathbf{2 a})$ & $C_{1}$ & -463.49296 & 0.012 & 8.5 & 7.5 \\
$\mathbf{2 a}$ & $C_{\mathrm{S}}$ & -463.50061 & 0.012 & 3.7 & 3.3 \\
$\mathbf{T S}(\mathbf{2 a} \rightarrow \mathbf{3})$ & $C_{\mathrm{S}}$ & -463.47821 & 0.012 & 17.8 & 17.2 \\
$\mathbf{3}$ & $C_{\mathrm{S}}$ & -463.52356 & 0.012 & -10.7 & -9.5 \\
\hline
\end{tabular}

a All relative energies are given relative to 1 . "rel E $(0 \mathrm{~K})$ " includes correction for differences in ZPE.

Table S4. M06-2X/cc-pVDZ Absolute Energies and ZPEs for $\mathrm{C}_{16} \mathrm{H}_{16}$ Stationary Points ${ }^{\mathrm{a}}$

\begin{tabular}{lcccccc}
\hline cpd & sym & $\begin{array}{c}\mathrm{E} \\
(\mathrm{au})\end{array}$ & $\begin{array}{c}\text { NIMAG } \\
\left(\omega, \mathrm{cm}^{-1}\right)\end{array}$ & $\begin{array}{c}\text { ZPE } \\
(\mathrm{kcal} / \mathrm{mol})\end{array}$ & $\begin{array}{c}\text { rel E, uncorr } \\
(\mathrm{kcal} / \mathrm{mol})\end{array}$ & $\begin{array}{c}\text { rel E }(0 \mathrm{~K}) \\
(\mathrm{kcal} / \mathrm{mol})\end{array}$ \\
\hline $\mathbf{4 a}$ & $S_{4}$ & -618.98435 & 0 & 169.19 & 0.0 & 0.0 \\
$\mathbf{T S}(\mathbf{4 a} \rightarrow \mathbf{4 c )})$ & $C_{1}$ & -618.96966 & $1(128 i)$ & 168.28 & 9.2 & 8.3 \\
$\mathbf{4 c}$ & $C_{1}$ & -618.97553 & 0 & 168.98 & 5.5 & 5.3 \\
$\mathbf{T S}(\mathbf{4 c} \rightarrow \mathbf{4 b})$ & $C_{1}$ & -618.97201 & $1(51 i)$ & 168.46 & 7.7 & 7.0 \\
$\mathbf{4 b}$ & $C_{1}$ & -618.97203 & 0 & 168.53 & 7.7 & 7.1 \\
$\mathbf{T S}(\mathbf{4 b \rightarrow 5 5})$ & $C_{1}$ & -618.95889 & $1(984 i)$ & 167.50 & 16.0 & 14.3 \\
$\mathbf{5 b}$ & $C_{1}$ & -618.97675 & 0 & 169.02 & 4.8 & 4.6 \\
$\mathbf{T S}(\mathbf{5 b} \rightarrow \mathbf{5 a})$ & $C_{1}$ & -618.97091 & $1(139 i)$ & 168.26 & 8.4 & 7.5 \\
$\mathbf{5 a}$ & $C_{1}$ & -618.98267 & 0 & 169.18 & 1.1 & 1.0 \\
\hline $\mathbf{A}$ & & & &
\end{tabular}

${ }^{a}$ All relative energies are given relative to 4a. "rel E $(0 \mathrm{~K})$ " includes correction for differences in ZPE. Species $\mathbf{4 c}$ is a conformational minimum between $\mathbf{4 a}$ and $\mathbf{4 b}$. 
Table S5. CCSD(T)/cc-pVDZ//M06-2X/cc-pVDZ Absolute Energies for $\mathrm{C}_{16} \mathrm{H}_{16}$ Stationary Points $^{\mathrm{a}}$

\begin{tabular}{lccccc}
\hline cpd & sym & $\begin{array}{c}\mathrm{CCSD}(\mathrm{T}) \\
\mathrm{E}(\mathrm{au})\end{array}$ & $\begin{array}{c}\mathrm{T}_{1} \\
\text { diagnostic }\end{array}$ & $\begin{array}{c}\text { rel E, uncorr } \\
(\mathrm{kcal} / \mathrm{mol})\end{array}$ & $\begin{array}{c}\text { rel E }(0 \mathrm{~K}) \\
(\mathrm{kcal} / \mathrm{mol})\end{array}$ \\
\hline $\mathbf{4 a}$ & $S_{4}$ & -617.44354 & 0.012 & 0.0 & 0.0 \\
$\mathbf{T S}(\mathbf{4 a} \rightarrow \mathbf{4 c})$ & $C_{1}$ & -617.43011 & 0.012 & 8.4 & 7.5 \\
$\mathbf{4 c}$ & $C_{1}$ & -617.43486 & 0.012 & 5.5 & 5.2 \\
$\mathbf{T S}(\mathbf{4 c} \rightarrow \mathbf{4 b})$ & $C_{1}$ & -617.43192 & 0.012 & 7.3 & 6.6 \\
$\mathbf{4 b}$ & $C_{1}$ & -617.43179 & 0.012 & 7.4 & 6.7 \\
$\mathbf{T S}(\mathbf{4 b} \rightarrow \mathbf{5 b})$ & $C_{1}$ & -617.41765 & 0.012 & 16.3 & 14.6 \\
$\mathbf{5 b}$ & $C_{1}$ & -617.43656 & 0.012 & 4.4 & 4.2 \\
$\mathbf{T S}(\mathbf{5 b} \rightarrow \mathbf{5 a})$ & $C_{1}$ & -617.43184 & 0.012 & 7.3 & 6.4 \\
$\mathbf{5 a}$ & $C_{1}$ & -617.44212 & 0.012 & 0.9 & 0.9 \\
\hline
\end{tabular}

a All relative energies are given relative to $4 a$. "rel E $(0 \mathrm{~K})$ " includes correction for differences in ZPE. Species $\mathbf{4 c}$ is a conformational minimum between $\mathbf{4 a}$ and $\mathbf{4 b}$.

\section{Note on Methods for Computing Tunneling Rate Constants}

One can, in principle, determine some details of thermally activated tunneling using Polyrate, by employing quantized reactant state tunneling (QRST). However, to do so, one must identify a vibrational mode in the reactant that corresponds to the reaction coordinate. For the annulene systems of interest here, this is a difficult task because the initial motion required is usually primarily conformational, whereas the event of primary interest is pi bond shifting. In our attempts to choose vibrational modes for use in QRST calculations, we have obtained unphysical results; for example, the computed rate constants with tunneling are lower than those without tunneling at some temperatures. As a result, we do not report QRST results and therefore cannot draw any conclusions about which vibrationally excited states are most relevant to the thermally activated tunneling in these systems. 


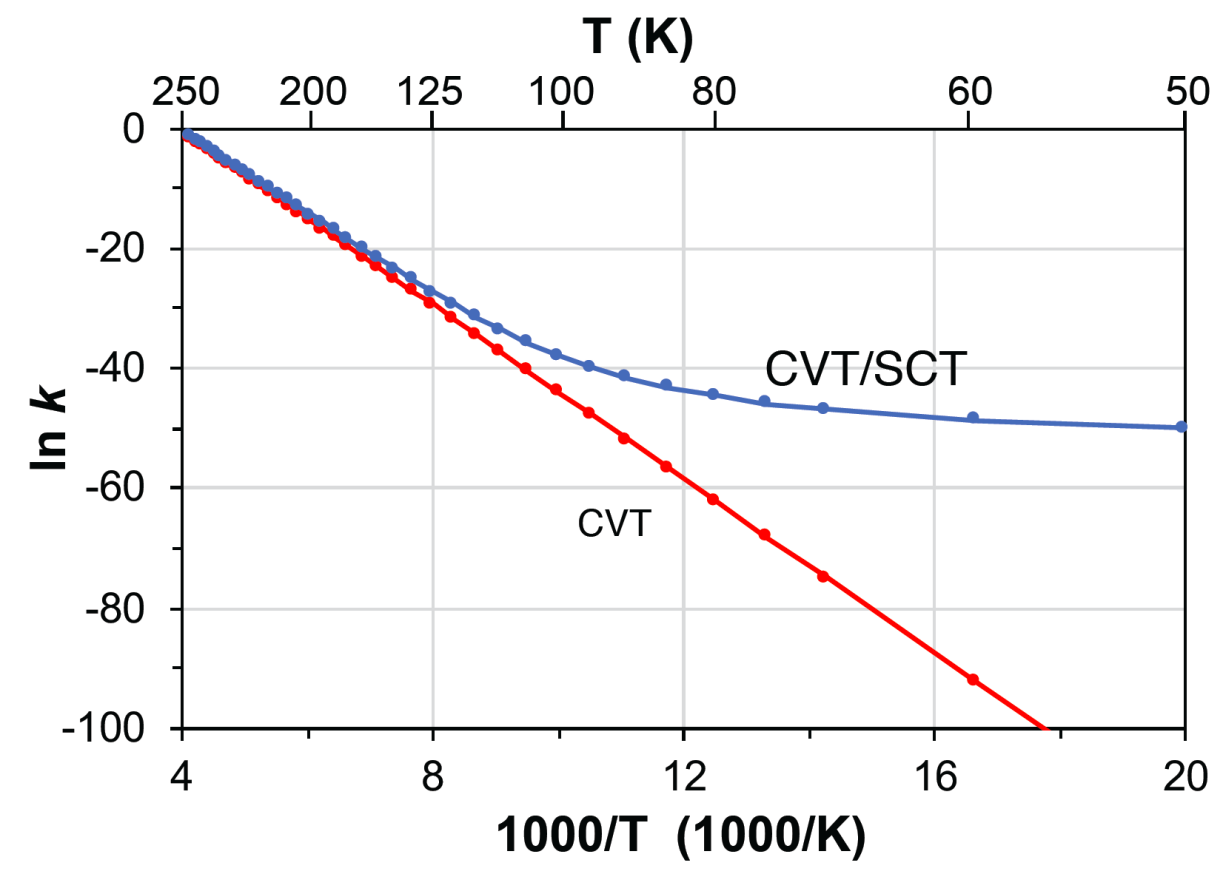

Figure S1. Arrhenius plot for 6-electron electrocyclic ring closure of CCCTCT-[12]annulene (2a) to bicyclic product 3. Rate constants obtained using the ISPE method, with the M06-2X/ccpVDZ potential energy surface adapted to $\mathrm{CCSD}(\mathrm{T}) / \mathrm{cc}-\mathrm{pVDZ}$ single point energies. Red $=\mathrm{CVT}$ $=$ over-barrier. Blue $=\mathrm{CVT} / \mathrm{SCT}$, i.e. including both over-barrier and tunneling contributions. 

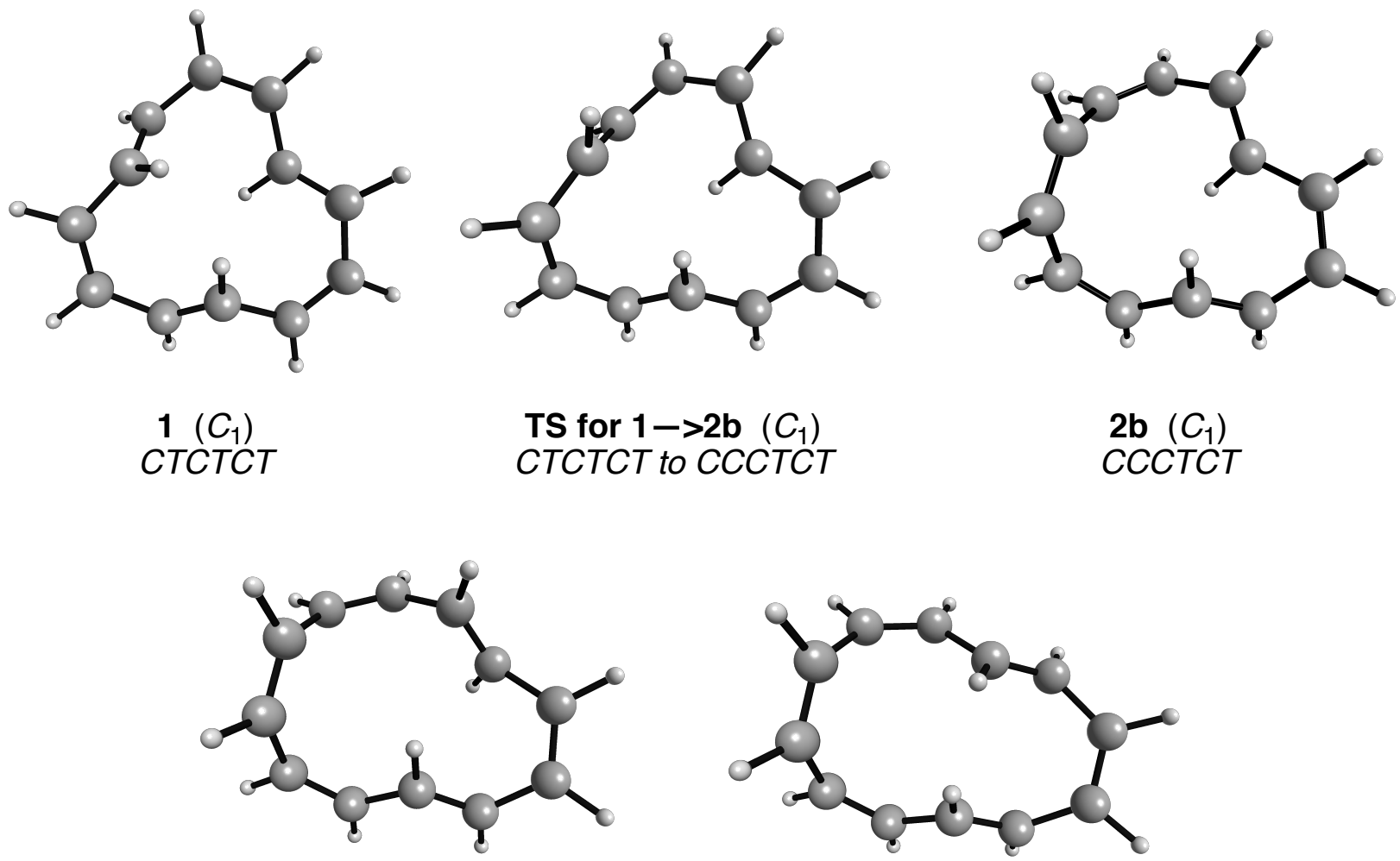

$\stackrel{2 b}{\text { C }}\left(C_{1}\right)$


TS for $2 \mathrm{a} \rightarrow \mathbf{3}\left(C_{\mathrm{s}}\right)$

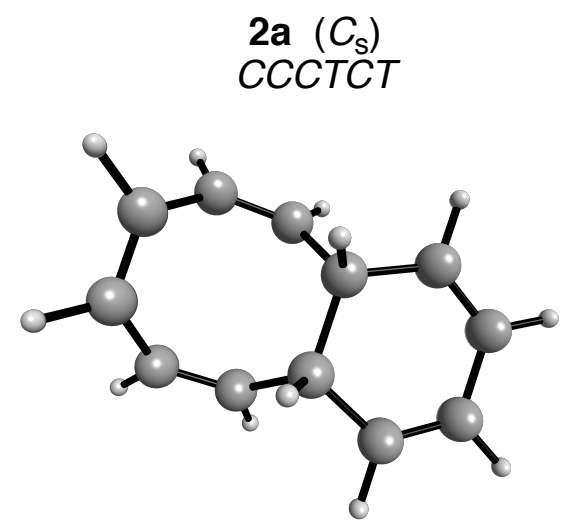

$3\left(C_{\mathrm{s}}\right)$

Figure S2. M06-2X/cc-pVDZ optimized geometries of stationary points involved in the complete mechanism for conversion of $\mathbf{1}$ to $\mathbf{3}$. 


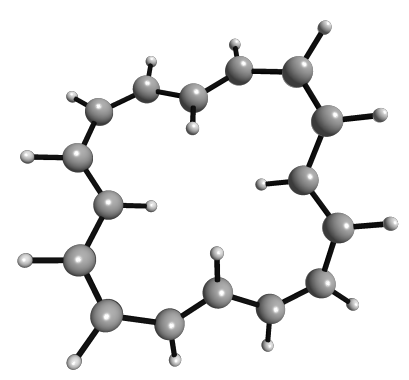

4a $\left(S_{4}\right)$ CTCTCTCT



TS $4 \mathrm{a} \rightarrow>\mathbf{4 c}\left(C_{1}\right)$ CTCTCTCT

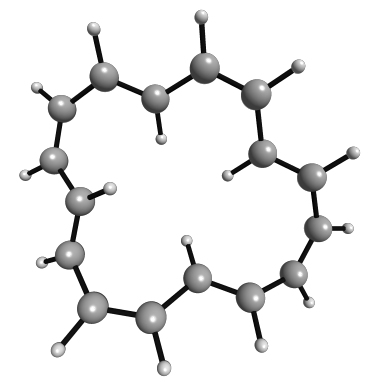

$\mathbf{4 c}\left(C_{1}\right)$
CTCTCTCT

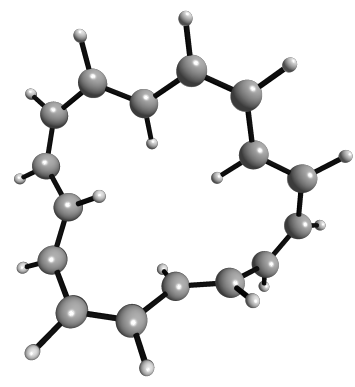

TS $4 \mathbf{c} \rightarrow \mathbf{4} \mathbf{b}\left(C_{1}\right)$

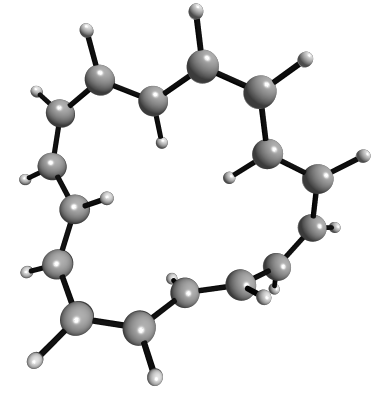

4b $\left(C_{1}\right)$ CTCTCTCT

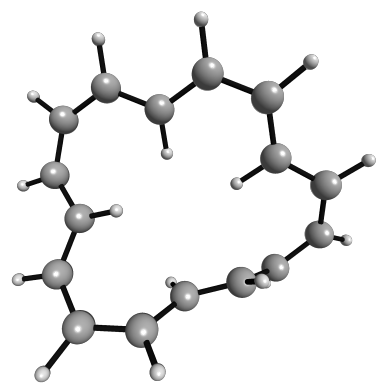

TS $\mathbf{4 b} \rightarrow \mathbf{b} \mathbf{b}\left(C_{1}\right)$ CTCTCTCT to CTCTTCTT

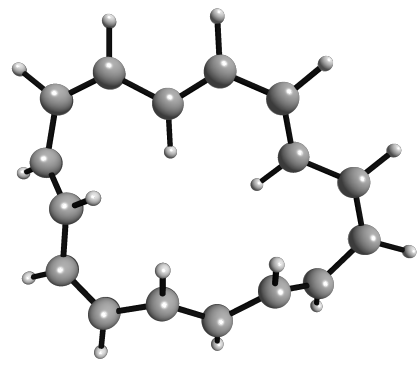

$5 \mathbf{b}\left(C_{1}\right)$ CTCTTCTT

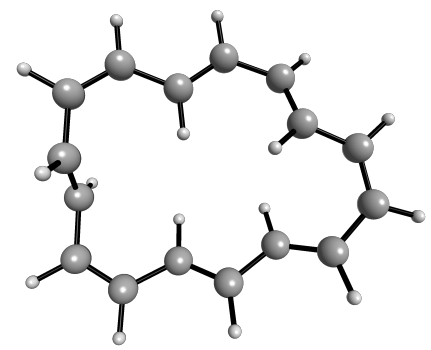

TS $5 \mathbf{b} \rightarrow \mathbf{5} \mathbf{a}\left(C_{1}\right)$ CTCTTCTT

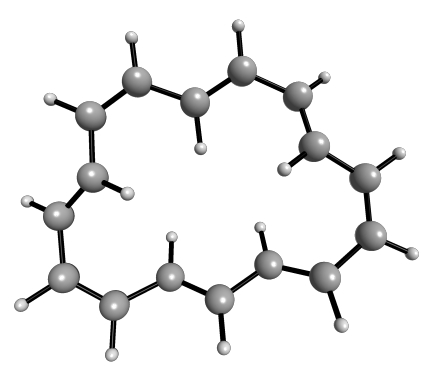

5a $\left(C_{1}\right)$ CTCTTCTT

Figure S3. M06-2X/cc-pVDZ optimized geometries of stationary points involved in the complete mechanism for conversion of $\mathbf{4 a}$ to $\mathbf{5 a}$. 

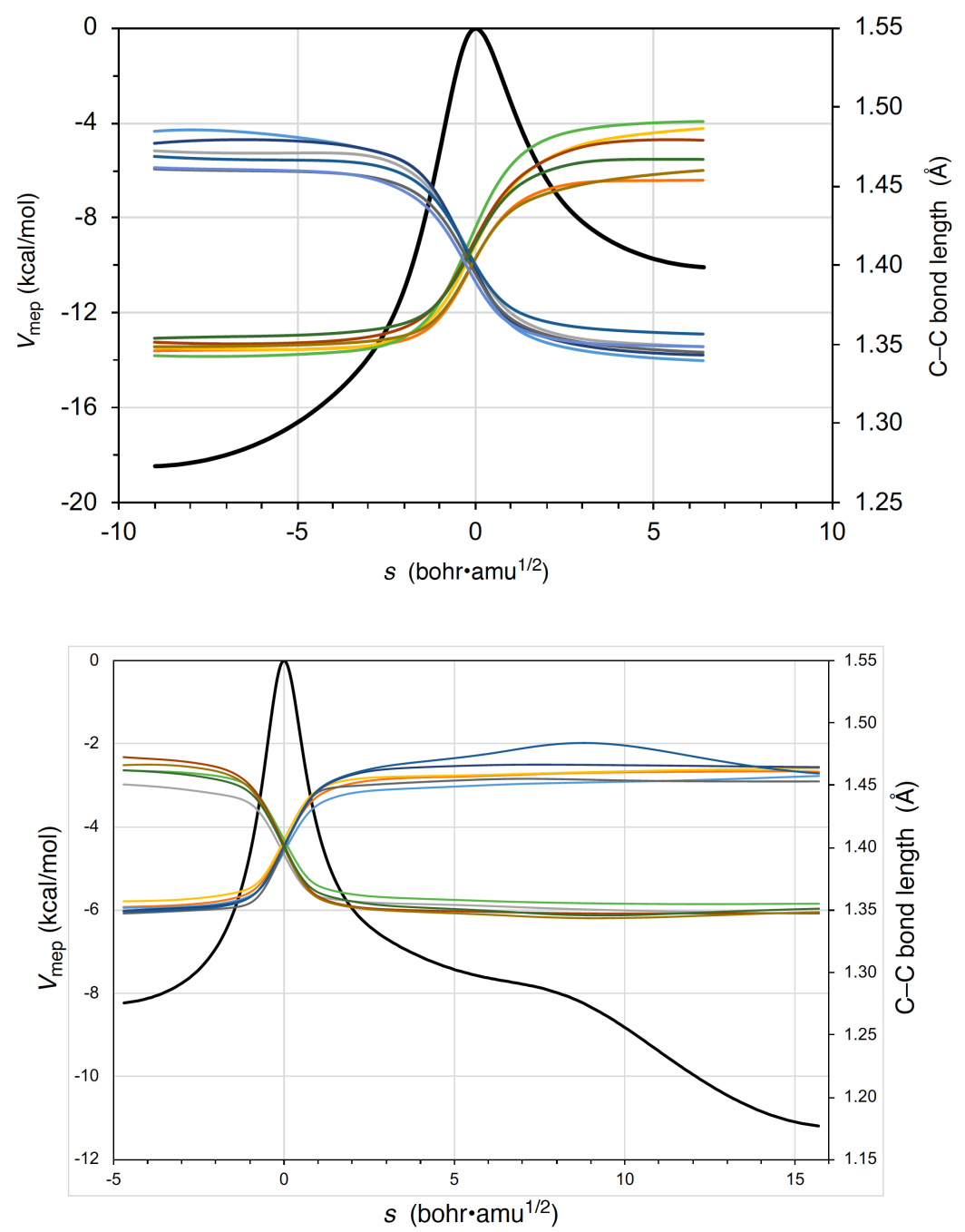

Figure S4. M06-2X/cc-pVDZ minimum-energy path curves as a function of mass-scaled reaction coordinate, showing changes in $\mathrm{C}-\mathrm{C}$ bond lengths along the reaction coordinate. Top: [12] annulene Möbius bond-shift step 1 to 2b. Bottom: [16]Annulene Möbius bond shifting from 4b to $\mathbf{5 b}$. In each plot, the thick black curve is the relative energy (with the transition state set to $0.0 \mathrm{kcal} / \mathrm{mol}$ ) and the other colored curves represent the $\mathrm{C}-\mathrm{C}$ bond lengths. 
Sample .dat (.fu5) file for Polyrate ISPE calculation of rate constants for the reaction $1 \rightarrow \mathbf{2 b}$ * GENERAL

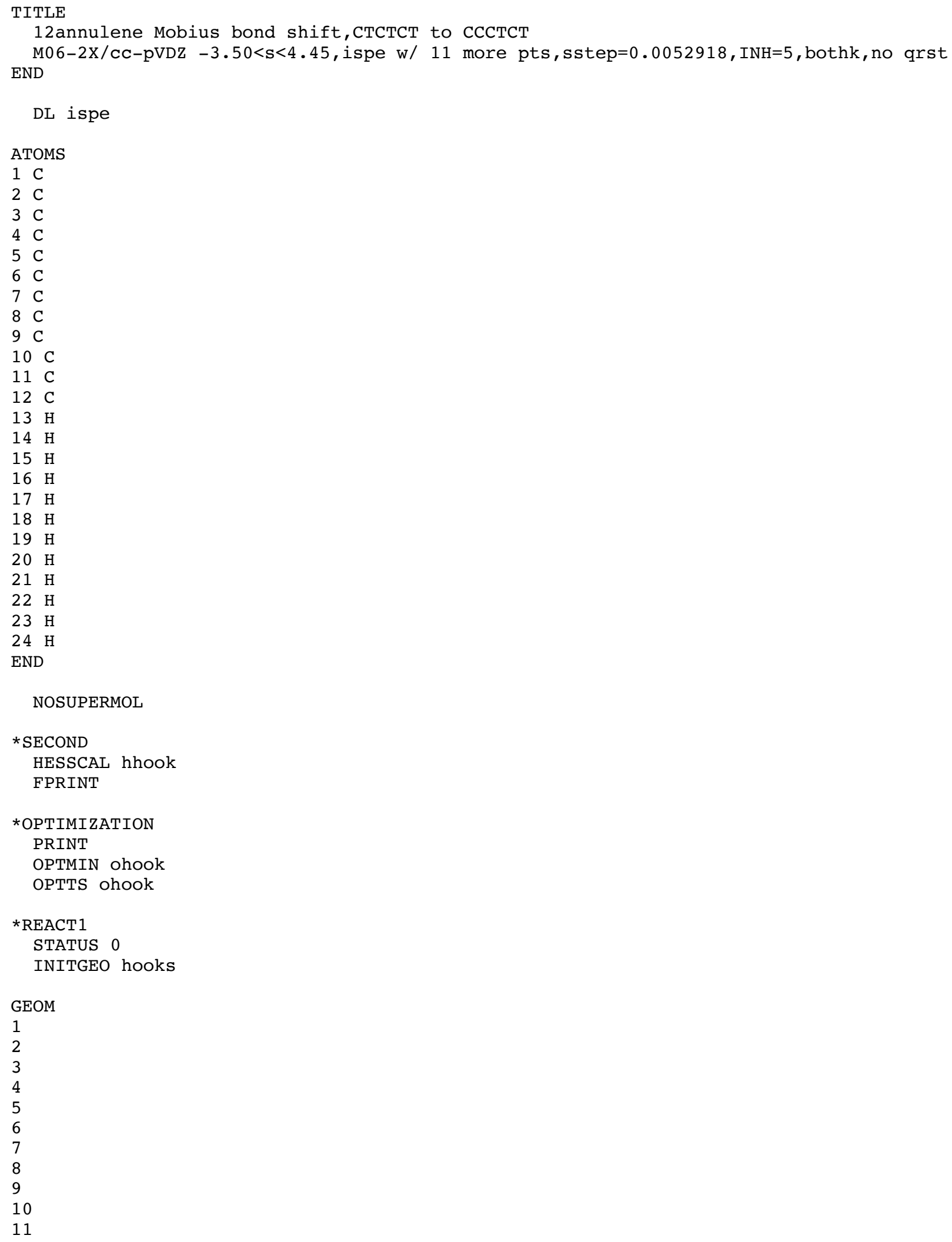


SPECIES nonlinrp

*PROD1 STATUS 0

INITGEO hooks

GEOM

1

2

3

4

5

6

7

8

10

11

12

13

14

15

16

17

18

19

20

21

22

23

24

END

SPECIES nonlinrp

*START

STATUS 0

INITGEO hooks

GEOM

1

2

3

4

5

6

7

8

10 
SPECIES nonlints

PROJECT

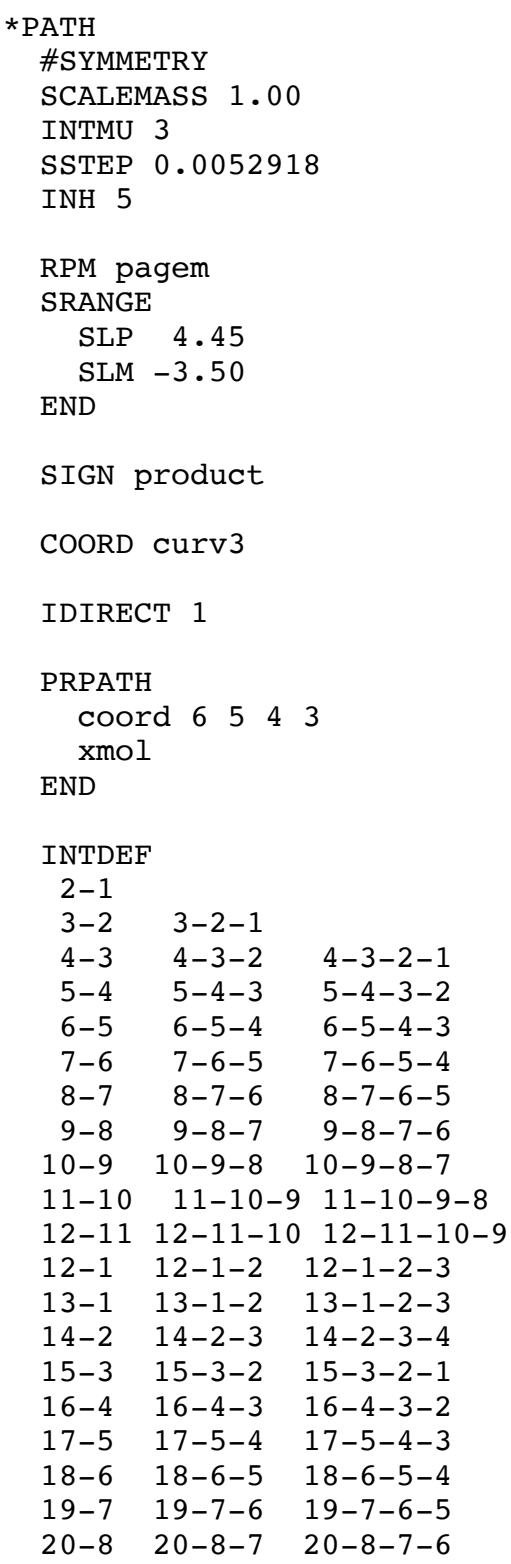







75.85.

95. 105 .

115. 125 .

135. 145 .

155. 165 .

175. 185 .

195. 205.

END

GTLOG

Sample .51 file for Polyrate ISPE calculation of rate constants for the reaction $\mathbf{4 b} \rightarrow \mathbf{5 b}$

*ISPEGEN MEPTYPER

MEPTYPEP

ENERXN 2.99

ENESAD 11.87

transition state

RCINFO

$\mathrm{SRC}$

ENERCS

ENERCA

END

PCINFO

SPC

ENEPCA 0.00 END
-4.95 \#S value of reactant (bohr)

\# unit fu51 input

\# reactant

\# product

\#classical energy difference between the reactant and the product \#CCSD(T) classical energy difference between the zero and the \#M06-2X/cc-pVDZ $\mathrm{E}$ of reactan \#CCSD(T)/cc-pVDZ $\mathrm{E}$ of reactant

\#S value of product (bohr) \#M06-2X/cc-pVDZ E of product \#CCSD ( T)/CC-pVDZ $\mathrm{E}$ of product 
Table S6. Computed Rate Constants for Möbius Bond Shifting in CTCTCT-[12]Annulene $(\mathbf{1} \rightarrow \mathbf{2 b})$, using ISPE method. CCSD(T)/cc-pVDZ//M06-2X/cc-pVDZ

\begin{tabular}{|c|c|c|c|c|c|}
\hline $\mathrm{T}(\mathrm{K})$ & $\begin{array}{c}\mathrm{k}(\mathrm{CVT}) \\
(\mathrm{s}-1)\end{array}$ & $\begin{array}{c}\mathrm{k}(\mathrm{CVT} / \mathrm{SCT}) \\
(\mathrm{s}-1)\end{array}$ & $\mathrm{T}(\mathrm{K})$ & $\begin{array}{c}\mathrm{k}(\mathrm{CVT}) \\
(\mathrm{s}-1)\end{array}$ & $\begin{array}{c}\mathrm{k}(\mathrm{CVT} / \mathrm{SCT}) \\
(\mathrm{s}-1)\end{array}$ \\
\hline 15 & $1.67 \mathrm{E}-255$ & $6.75 \mathrm{E}-122$ & 148 & $1.44 \mathrm{E}-15$ & $2.26 \mathrm{E}-14$ \\
\hline 18 & $4.73 E-211$ & $6.09 \mathrm{E}-104$ & 150 & $3.32 E-15$ & $4.65 E-14$ \\
\hline 20 & $8.07 E-189$ & $6.11 \mathrm{E}-95$ & 152 & $7.45 \mathrm{E}-15$ & $9.40 \mathrm{E}-14$ \\
\hline 22 & $1.26 \mathrm{E}-170$ & $1.47 \mathrm{E}-87$ & 155 & $2.41 \mathrm{E}-14$ & $2.63 E-13$ \\
\hline 25 & $8.65 \mathrm{E}-149$ & $1.15 \mathrm{E}-78$ & 158 & $7.46 \mathrm{E}-14$ & $7.14 \mathrm{E}-13$ \\
\hline 28 & $1.26 \mathrm{E}-131$ & 1. $22 \mathrm{E}-71$ & 160 & $1.55 \mathrm{E}-13$ & $1.36 \mathrm{E}-12$ \\
\hline 30 & $4.32 \mathrm{E}-122$ & $1.03 E-67$ & 162 & $3.15 \mathrm{E}-13$ & $2.57 \mathrm{E}-12$ \\
\hline 32 & $9.57 \mathrm{E}-114$ & $2.92 E-64$ & 165 & $8.88 E-13$ & $6.51 \mathrm{E}-12$ \\
\hline 35 & $5.16 \mathrm{E}-103$ & $8.72 \mathrm{E}-60$ & 170 & $4.60 \mathrm{E}-12$ & $2.87 \mathrm{E}-11$ \\
\hline 38 & $5.64 \mathrm{E}-94$ & $5.65 \mathrm{E}-56$ & 175 & $2.17 \mathrm{E}-11$ & $1.18 \mathrm{E}-10$ \\
\hline 40 & $1.06 \mathrm{E}-88$ & $9.97 \mathrm{E}-54$ & 180 & $9.36 \mathrm{E}-11$ & $4.49 \mathrm{E}-10$ \\
\hline 42 & $6.24 \mathrm{E}-84$ & 1. $12 \mathrm{E}-51$ & 185 & $3.74 \mathrm{E}-10$ & $1.61 \mathrm{E}-09$ \\
\hline 45 & $1.44 \mathrm{E}-77$ & $6.63 E-49$ & 190 & $1.39 E-09$ & $5.41 \mathrm{E}-09$ \\
\hline 48 & $5.31 E-72$ & $1.93 E-46$ & 195 & $4.81 E-09$ & $1.72 \mathrm{E}-08$ \\
\hline 50 & $1.16 \mathrm{E}-68$ & $6.11 \mathrm{E}-45$ & 200 & $1.57 \mathrm{E}-08$ & $5.19 E-08$ \\
\hline 52 & $1.41 \mathrm{E}-65$ & $1.54 \mathrm{E}-43$ & 205 & $4.83 E-08$ & $1.49 \mathrm{E}-07$ \\
\hline 55 & $2.26 \mathrm{E}-61$ & 1. $34 \mathrm{E}-41$ & 210 & $1.41 \mathrm{E}-07$ & $4.07 \mathrm{E}-07$ \\
\hline 58 & $1.33 \mathrm{E}-57$ & $7.93 \mathrm{E}-40$ & 215 & $3.91 \mathrm{E}-07$ & $1.06 \mathrm{E}-06$ \\
\hline 60 & $2.69 \mathrm{E}-55$ & $1.00 \mathrm{E}-38$ & 220 & $1.04 \mathrm{E}-06$ & $2.67 \mathrm{E}-06$ \\
\hline 62 & $3.85 E-53$ & $1.11 \mathrm{E}-37$ & 225 & $2.63 E-06$ & $6.45 E-06$ \\
\hline 65 & $3.72 E-50$ & $3.26 \mathrm{E}-36$ & 230 & $6.40 \mathrm{E}-06$ & $1.50 \mathrm{E}-05$ \\
\hline 68 & $1.96 \mathrm{E}-47$ & $7.63 E-35$ & 233 & $1.07 \mathrm{E}-05$ & $2.45 \mathrm{E}-05$ \\
\hline 70 & $9.47 \mathrm{E}-46$ & $5.56 \mathrm{E}-34$ & 235 & $1.50 \mathrm{E}-05$ & $3.38 \mathrm{E}-05$ \\
\hline 72 & $3.69 \mathrm{E}-44$ & $3.73 E-33$ & 240 & $3.40 E-05$ & $7.35 \mathrm{E}-05$ \\
\hline 75 & $6.24 E-42$ & $5.63 E-32$ & 245 & $7.43 E-05$ & $1.55 \mathrm{E}-04$ \\
\hline 78 & $7.10 \mathrm{E}-40$ & $7.30 E-31$ & 250 & $1.58 \mathrm{E}-04$ & $3.19 \mathrm{E}-04$ \\
\hline 80 & $1.37 \mathrm{E}-38$ & $3.74 \mathrm{E}-30$ & 255 & $3.25 E-04$ & $6.36 \mathrm{E}-04$ \\
\hline 82 & $2.29 \mathrm{E}-37$ & $1.81 \mathrm{E}-29$ & 260 & $6.50 \mathrm{E}-04$ & $1.24 \mathrm{E}-03$ \\
\hline 85 & 1. $22 \mathrm{E}-35$ & $1.75 \mathrm{E}-28$ & 265 & $1.27 \mathrm{E}-03$ & $2.35 \mathrm{E}-03$ \\
\hline 88 & $4.93 E-34$ & $1.53 \mathrm{E}-27$ & 270 & $2.42 \mathrm{E}-03$ & $4.36 \mathrm{E}-03$ \\
\hline 90 & $5.07 E-33$ & $6.14 \mathrm{E}-27$ & 273 & $3.51 E-03$ & $6.26 \mathrm{E}-03$ \\
\hline 92 & $4.72 E-32$ & $2.37 E-26$ & 275 & $4.49 E-03$ & $7.93 E-03$ \\
\hline 95 & $1.12 \mathrm{E}-30$ & $1.68 \mathrm{E}-25$ & 280 & $8.17 E-03$ & $1.41 \mathrm{E}-02$ \\
\hline 98 & $2.20 \mathrm{E}-29$ & $1.10 \mathrm{E}-24$ & 285 & $1.45 \mathrm{E}-02$ & $2.46 \mathrm{E}-02$ \\
\hline 100 & 1. $45 \mathrm{E}-28$ & 3. $72 \mathrm{E}-24$ & 290 & $2.54 \mathrm{E}-02$ & $4.21 \mathrm{E}-02$ \\
\hline 102 & $8.84 E-28$ & $1.22 \mathrm{E}-23$ & 293 & $3.52 E-02$ & $5.76 \mathrm{E}-02$ \\
\hline 105 & $1.17 \mathrm{E}-26$ & $6.85 E-23$ & 295 & $4.35 E-02$ & $7.08 E-02$ \\
\hline 108 & $1.35 \mathrm{E}-25$ & $3.64 \mathrm{E}-22$ & 298 & $5.96 \mathrm{E}-02$ & $9.59 \mathrm{E}-02$ \\
\hline 110 & $6.38 E-25$ & $1.07 \mathrm{E}-21$ & 300 & $7.32 \mathrm{E}-02$ & $1.17 \mathrm{E}-01$ \\
\hline 112 & $2.85 E-24$ & $3.09 E-21$ & 310 & $1.97 E-01$ & $3.05 \mathrm{E}-01$ \\
\hline 115 & $2.45 E-23$ & $1.45 \mathrm{E}-20$ & 320 & $4.99 \mathrm{E}-01$ & $7.50 \mathrm{E}-01$ \\
\hline 118 & 1. $89 \mathrm{E}-22$ & $6.52 \mathrm{E}-20$ & 330 & $1.19 \mathrm{E}+00$ & $1.75 \mathrm{E}+00$ \\
\hline 120 & $6.94 \mathrm{E}-22$ & $1.73 E-19$ & 340 & $2.71 E+00$ & $3.88 \mathrm{E}+00$ \\
\hline 122 & $2.45 E-21$ & $4.50 \mathrm{E}-19$ & 350 & $5.88 E+00$ & $8.23 E+00$ \\
\hline 125 & $1.50 \mathrm{E}-20$ & $1.82 \mathrm{E}-18$ & 360 & $1.22 \mathrm{E}+01$ & $1.68 \mathrm{E}+01$ \\
\hline 128 & $8.49 \mathrm{E}-20$ & $7.08 \mathrm{E}-18$ & 370 & $2.44 \mathrm{E}+01$ & 3. $29 \mathrm{E}+01$ \\
\hline 130 & $2.57 \mathrm{E}-19$ & $1.71 \mathrm{E}-17$ & 380 & $4.70 E+01$ & $6.23 E+01$ \\
\hline 132 & $7.55 \mathrm{E}-19$ & $4.06 \mathrm{E}-17$ & 390 & $8.75 E+01$ & $1.14 \mathrm{E}+02$ \\
\hline 135 & $3.57 \mathrm{E}-18$ & $1.44 \mathrm{E}-16$ & 400 & $1.58 \mathrm{E}+02$ & $2.04 \mathrm{E}+02$ \\
\hline 138 & $1.58 \mathrm{E}-17$ & $4.91 \mathrm{E}-16$ & & & \\
\hline 140 & $4.10 \mathrm{E}-17$ & $1.09 \mathrm{E}-15$ & & & \\
\hline 142 & $1.04 \mathrm{E}-16$ & $2.38 E-15$ & & & \\
\hline 145 & $3.98 \mathrm{E}-16$ & $7.47 \mathrm{E}-15$ & & & \\
\hline
\end{tabular}


Table S7. Computed Rate Constants for 6-Electron Electrocyclic Ring Closure of CCCTCT[12]Annulene $(\mathbf{2 a} \rightarrow \mathbf{3})$, using ISPE method. CCSD(T)/cc-pVDZ//M06-2X/cc-pVDZ

\begin{tabular}{|c|c|c|c|c|c|}
\hline $\mathrm{T}(\mathrm{K})$ & $\begin{array}{c}\mathrm{k}(\mathrm{CVT}) \\
(\mathrm{s}-1)\end{array}$ & $\begin{array}{c}\mathrm{k}(\mathrm{CVT} / \mathrm{SCT}) \\
(\mathrm{s}-1)\end{array}$ & $\mathrm{T}(\mathrm{K})$ & $\begin{array}{c}\mathrm{k}(\mathrm{CVT}) \\
(\mathrm{s}-1)\end{array}$ & $\begin{array}{c}\mathrm{k}(\mathrm{CVT} / \mathrm{SCT}) \\
(\mathrm{s}-1)\end{array}$ \\
\hline 40 & $5.91 \mathrm{E}-67$ & $8.37 \mathrm{E}-23$ & 278 & $1.04 \mathrm{E}+01$ & $1.40 \mathrm{E}+01$ \\
\hline 50 & $3.02 \mathrm{E}-51$ & $2.12 \mathrm{E}-22$ & 280 & $1.26 \mathrm{E}+01$ & $1.68 \mathrm{E}+01$ \\
\hline 60 & $9.13 \mathrm{E}-41$ & $7.26 \mathrm{E}-22$ & 282 & $1.51 \mathrm{E}+01$ & $2.01 \mathrm{E}+01$ \\
\hline 70 & $2.82 \mathrm{E}-33$ & $4.04 \mathrm{E}-21$ & 290 & $3.04 \mathrm{E}+01$ & $3.98 \mathrm{E}+01$ \\
\hline 75 & $2.80 \mathrm{E}-30$ & $1.20 \mathrm{E}-20$ & 293 & $3.92 \mathrm{E}+01$ & $5.10 \mathrm{E}+01$ \\
\hline 80 & $1.17 \mathrm{E}-27$ & $4.24 \mathrm{E}-20$ & 296 & $5.02 \mathrm{E}+01$ & $6.50 \mathrm{E}+01$ \\
\hline 85 & $2.41 \mathrm{E}-25$ & $1.78 \mathrm{E}-19$ & 298 & $5.90 \mathrm{E}+01$ & $7.62 \mathrm{E}+01$ \\
\hline 90 & $2.75 \mathrm{E}-23$ & $8.83 E-19$ & 300 & $6.93 E+01$ & $8.92 \mathrm{E}+01$ \\
\hline 95 & $1.90 \mathrm{E}-21$ & $5.17 \mathrm{E}-18$ & 302 & $8.12 \mathrm{E}+01$ & $1.04 \mathrm{E}+02$ \\
\hline 100 & $8.61 \mathrm{E}-20$ & $3.55 \mathrm{E}-17$ & & & \\
\hline 105 & $2.71 \mathrm{E}-18$ & $2.80 \mathrm{E}-16$ & & & \\
\hline 110 & $6.23 \mathrm{E}-17$ & $2.42 \mathrm{E}-15$ & & & \\
\hline 115 & $1.09 \mathrm{E}-15$ & $2.14 \mathrm{E}-14$ & & & \\
\hline 120 & $1.50 \mathrm{E}-14$ & $1.82 \mathrm{E}-13$ & & & \\
\hline 125 & $1.68 \mathrm{E}-13$ & $1.43 \mathrm{E}-12$ & & & \\
\hline 130 & $1.55 \mathrm{E}-12$ & $1.01 \mathrm{E}-11$ & & & \\
\hline 135 & $1.22 \mathrm{E}-11$ & $6.42 \mathrm{E}-11$ & & & \\
\hline 140 & $8.26 \mathrm{E}-11$ & $3.66 \mathrm{E}-10$ & & & \\
\hline 145 & $4.90 \mathrm{E}-10$ & $1.89 \mathrm{E}-09$ & & & \\
\hline 150 & $2.58 \mathrm{E}-09$ & $8.81 \mathrm{E}-09$ & & & \\
\hline 155 & $1.22 \mathrm{E}-08$ & $3.76 \mathrm{E}-08$ & & & \\
\hline 160 & $5.25 \mathrm{E}-08$ & $1.48 \mathrm{E}-07$ & & & \\
\hline 165 & $2.06 \mathrm{E}-07$ & $5.37 \mathrm{E}-07$ & & & \\
\hline 170 & $7.46 \mathrm{E}-07$ & $1.82 \mathrm{E}-06$ & & & \\
\hline 175 & $2.51 \mathrm{E}-06$ & $5.76 \mathrm{E}-06$ & & & \\
\hline 180 & $7.89 \mathrm{E}-06$ & $1.71 \mathrm{E}-05$ & & & \\
\hline 185 & $2.33 \mathrm{E}-05$ & $4.82 \mathrm{E}-05$ & & & \\
\hline 190 & $6.50 \mathrm{E}-05$ & $1.29 \mathrm{E}-04$ & & & \\
\hline 195 & $1.72 \mathrm{E}-04$ & $3.28 \mathrm{E}-04$ & & & \\
\hline 198 & $3.01 \mathrm{E}-04$ & $5.61 \mathrm{E}-04$ & & & \\
\hline 200 & $4.33 \mathrm{E}-04$ & $7.96 \mathrm{E}-04$ & & & \\
\hline 202 & $6.19 \mathrm{E}-04$ & $1.12 \mathrm{E}-03$ & & & \\
\hline 210 & $2.41 \mathrm{E}-03$ & $4.15 \mathrm{E}-03$ & & & \\
\hline 218 & $8.48 \mathrm{E}-03$ & $1.40 \mathrm{E}-02$ & & & \\
\hline 220 & $1.14 \mathrm{E}-02$ & $1.87 \mathrm{E}-02$ & & & \\
\hline 222 & $1.54 \mathrm{E}-02$ & $2.49 \mathrm{E}-02$ & & & \\
\hline 230 & $4.75 \mathrm{E}-02$ & $7.41 \mathrm{E}-02$ & & & \\
\hline 231 & $5.43 \mathrm{E}-02$ & $8.44 \mathrm{E}-02$ & & & \\
\hline 233 & $7.10 \mathrm{E}-02$ & $1.09 \mathrm{E}-01$ & & & \\
\hline 235 & $9.23 \mathrm{E}-02$ & $1.41 \mathrm{E}-01$ & & & \\
\hline 238 & $1.36 \mathrm{E}-01$ & $2.05 \mathrm{E}-01$ & & & \\
\hline 240 & $1.75 \mathrm{E}-01$ & $2.62 \mathrm{E}-01$ & & & \\
\hline 242 & $2.24 \mathrm{E}-01$ & $3.33 \mathrm{E}-01$ & & & \\
\hline 248 & $4.59 \mathrm{E}-01$ & $6.70 \mathrm{E}-01$ & & & \\
\hline 250 & $5.79 \mathrm{E}-01$ & $8.39 \mathrm{E}-01$ & & & \\
\hline 252 & $7.27 \mathrm{E}-01$ & $1.05 \mathrm{E}+00$ & & & \\
\hline 258 & $1.41 \mathrm{E}+00$ & $2.00 \mathrm{E}+00$ & & & \\
\hline 260 & $1.75 \mathrm{E}+00$ & $2.46 \mathrm{E}+00$ & & & \\
\hline 262 & $2.16 \mathrm{E}+00$ & $3.02 \mathrm{E}+00$ & & & \\
\hline 270 & $4.86 \mathrm{E}+00$ & $6.66 \mathrm{E}+00$ & & & \\
\hline 271 & $5.36 \mathrm{E}+00$ & $7.33 E+00$ & & & \\
\hline 273 & $6.51 \mathrm{E}+00$ & $8.85 E+00$ & & & \\
\hline 275 & $7.88 \mathrm{E}+00$ & $1.07 \mathrm{E}+01$ & & & \\
\hline
\end{tabular}


Table S8. Computed Rate Constants for Möbius Bond Shift in CTCTCTCT-[16]Annulene $(\mathbf{4 b} \rightarrow \mathbf{5 b})$ using ISPE method. (CCSD $(\mathrm{T})$ energies mapped onto M06-2X potential surface)

\begin{tabular}{|c|c|c|c|c|c|}
\hline $\mathrm{T}(\mathrm{K})$ & $\begin{array}{c}\mathrm{k}(\mathrm{CVT}) \\
(\mathrm{s}-1)\end{array}$ & $\begin{array}{c}\mathrm{k}(\mathrm{CVT} / \mathrm{SCT}) \\
(\mathrm{s}-1)\end{array}$ & $\mathrm{T}(\mathrm{K})$ & $\begin{array}{c}\mathrm{k}(\mathrm{CVT}) \\
(\mathrm{s}-1)\end{array}$ & $\begin{array}{c}\mathrm{k}(\mathrm{CVT} / \mathrm{SCT}) \\
(\mathrm{s}-1)\end{array}$ \\
\hline 25 & $1.15 \mathrm{E}-57$ & $2.33 \mathrm{E}-09$ & 158 & $1.39 \mathrm{E}+01$ & $1.22 \mathrm{E}+03$ \\
\hline 28 & $2.83 \mathrm{E}-50$ & $1.30 \mathrm{E}-08$ & 160 & $1.90 \mathrm{E}+01$ & $1.46 \mathrm{E}+03$ \\
\hline 30 & $3.63 \mathrm{E}-46$ & $3.75 \mathrm{E}-08$ & 162 & $2.58 \mathrm{E}+01$ & $1.74 \mathrm{E}+03$ \\
\hline 32 & $1.43 \mathrm{E}-42$ & $1.01 \mathrm{E}-07$ & 165 & $4.02 \mathrm{E}+01$ & $2.25 E+03$ \\
\hline 35 & $6.00 \mathrm{E}-38$ & $3.97 \mathrm{E}-07$ & 170 & $8.14 \mathrm{E}+01$ & $3.42 \mathrm{E}+03$ \\
\hline 38 & $4.70 \mathrm{E}-34$ & $1.39 \mathrm{E}-06$ & 175 & $1.58 \mathrm{E}+02$ & $5.14 \mathrm{E}+03$ \\
\hline 40 & $8.77 \mathrm{E}-32$ & $3.04 \mathrm{E}-06$ & 180 & $2.97 \mathrm{E}+02$ & $7.62 \mathrm{E}+03$ \\
\hline 42 & $9.95 \mathrm{E}-30$ & $6.38 \mathrm{E}-06$ & 185 & $5.38 \mathrm{E}+02$ & $1.12 \mathrm{E}+04$ \\
\hline 45 & $5.47 \mathrm{E}-27$ & $1.81 \mathrm{E}-05$ & 190 & $9.46 \mathrm{E}+02$ & $1.62 \mathrm{E}+04$ \\
\hline 48 & $1.37 \mathrm{E}-24$ & $4.78 \mathrm{E}-05$ & 195 & $1.61 \mathrm{E}+03$ & $2.31 \mathrm{E}+04$ \\
\hline 50 & $3.75 \mathrm{E}-23$ & $8.81 \mathrm{E}-05$ & 200 & $2.68 \mathrm{E}+03$ & $3.27 E+04$ \\
\hline 52 & $7.99 \mathrm{E}-22$ & $1.58 \mathrm{E}-04$ & 205 & $4.35 E+03$ & $4.58 \mathrm{E}+04$ \\
\hline 55 & $5.16 \mathrm{E}-20$ & $3.64 \mathrm{E}-04$ & 210 & $6.89 \mathrm{E}+03$ & $6.34 \mathrm{E}+04$ \\
\hline 58 & $2.17 \mathrm{E}-18$ & $7.98 \mathrm{E}-04$ & 215 & $1.07 \mathrm{E}+04$ & $8.69 E+04$ \\
\hline 60 & $2.13 \mathrm{E}-17$ & $1.31 \mathrm{E}-03$ & 220 & $1.62 \mathrm{E}+04$ & $1.18 \mathrm{E}+05$ \\
\hline 62 & $1.80 \mathrm{E}-16$ & $2.12 \mathrm{E}-03$ & 225 & $2.42 \mathrm{E}+04$ & $1.59 \mathrm{E}+05$ \\
\hline 65 & $3.47 \mathrm{E}-15$ & $4.23 \mathrm{E}-03$ & 230 & $3.55 \mathrm{E}+04$ & $2.11 \mathrm{E}+05$ \\
\hline 68 & $5.15 \mathrm{E}-14$ & $8.15 \mathrm{E}-03$ & 235 & $5.13 E+04$ & $2.79 E+05$ \\
\hline 70 & $2.73 \mathrm{E}-13$ & $1.24 \mathrm{E}-02$ & 240 & $7.29 \mathrm{E}+04$ & $3.65 E+05$ \\
\hline 72 & $1.32 \mathrm{E}-12$ & $1.86 \mathrm{E}-02$ & 245 & $1.02 \mathrm{E}+05$ & $4.73 E+05$ \\
\hline 75 & $1.20 \mathrm{E}-11$ & $3.35 \mathrm{E}-02$ & 250 & $1.41 \mathrm{E}+05$ & $6.09 \mathrm{E}+05$ \\
\hline 78 & $9.19 \mathrm{E}-11$ & $5.87 \mathrm{E}-02$ & 255 & $1.93 E+05$ & $7.78 \mathrm{E}+05$ \\
\hline 80 & $3.28 \mathrm{E}-10$ & $8.43 \mathrm{E}-02$ & 260 & $2.60 \mathrm{E}+05$ & $9.86 \mathrm{E}+05$ \\
\hline 82 & $1.10 \mathrm{E}-09$ & $1.20 \mathrm{E}-01$ & 265 & $3.46 \mathrm{E}+05$ & $1.24 \mathrm{E}+06$ \\
\hline 85 & $6.08 \mathrm{E}-09$ & $2.00 \mathrm{E}-01$ & 270 & $4.57 E+05$ & $1.55 \mathrm{E}+06$ \\
\hline 88 & $2.99 \mathrm{E}-08$ & $3.27 \mathrm{E}-01$ & 273 & $5.37 \mathrm{E}+05$ & $1.77 \mathrm{E}+06$ \\
\hline 90 & $8.13 \mathrm{E}-08$ & $4.50 \mathrm{E}-01$ & 275 & $5.97 \mathrm{E}+05$ & $1.93 E+06$ \\
\hline 92 & $2.12 \mathrm{E}-07$ & $6.14 \mathrm{E}-01$ & 280 & $7.73 E+05$ & $2.38 E+06$ \\
\hline 95 & $8.28 \mathrm{E}-07$ & $9.65 \mathrm{E}-01$ & 285 & $9.91 \mathrm{E}+05$ & $2.92 \mathrm{E}+06$ \\
\hline 98 & $2.97 \mathrm{E}-06$ & $1.50 \mathrm{E}+00$ & 290 & $1.26 \mathrm{E}+06$ & $3.56 \mathrm{E}+06$ \\
\hline 100 & $6.68 \mathrm{E}-06$ & $1.99 \mathrm{E}+00$ & 293 & $1.45 \mathrm{E}+06$ & $4.00 \mathrm{E}+06$ \\
\hline 102 & $1.45 \mathrm{E}-05$ & $2.63 \mathrm{E}+00$ & 295 & $1.59 \mathrm{E}+06$ & $4.32 E+06$ \\
\hline 105 & $4.41 \mathrm{E}-05$ & $3.95 \mathrm{E}+00$ & 298 & $1.82 \mathrm{E}+06$ & $4.83 E+06$ \\
\hline 108 & $1.26 \mathrm{E}-04$ & $5.86 \mathrm{E}+00$ & 300 & $1.99 \mathrm{E}+06$ & $5.20 \mathrm{E}+06$ \\
\hline 110 & $2.46 \mathrm{E}-04$ & $7.58 \mathrm{E}+00$ & 310 & $3.05 E+06$ & $7.45 \mathrm{E}+06$ \\
\hline 112 & $4.67 \mathrm{E}-04$ & $9.76 \mathrm{E}+00$ & 320 & $4.55 E+06$ & $1.05 E+07$ \\
\hline 115 & $1.18 \mathrm{E}-03$ & $1.41 \mathrm{E}+01$ & 330 & $6.63 E+06$ & $1.44 \mathrm{E}+07$ \\
\hline 118 & $2.83 \mathrm{E}-03$ & $2.03 E+01$ & 340 & $9.45 E+06$ & $1.96 \mathrm{E}+07$ \\
\hline 120 & $4.95 \mathrm{E}-03$ & $2.57 \mathrm{E}+01$ & 350 & $1.32 \mathrm{E}+07$ & $2.62 E+07$ \\
\hline 122 & $8.50 \mathrm{E}-03$ & $3.23 E+01$ & 360 & $1.81 \mathrm{E}+07$ & $3.44 \mathrm{E}+07$ \\
\hline 125 & $1.85 \mathrm{E}-02$ & $4.54 \mathrm{E}+01$ & 370 & $2.44 E+07$ & $4.47 E+07$ \\
\hline 128 & $3.90 \mathrm{E}-02$ & $6.34 E+01$ & 380 & $3.24 E+07$ & $5.74 \mathrm{E}+07$ \\
\hline 130 & $6.27 \mathrm{E}-02$ & $7.88 \mathrm{E}+01$ & 390 & $4.24 E+07$ & $7.28 \mathrm{E}+07$ \\
\hline 132 & $9.95 \mathrm{E}-02$ & $9.76 \mathrm{E}+01$ & 400 & $5.48 \mathrm{E}+07$ & $9.14 E+07$ \\
\hline 135 & $1.94 \mathrm{E}-01$ & $1.34 \mathrm{E}+02$ & & & \\
\hline 138 & $3.67 \mathrm{E}-01$ & $1.82 \mathrm{E}+02$ & & & \\
\hline 140 & $5.53 \mathrm{E}-01$ & $2.23 E+02$ & & & \\
\hline 142 & $8.24 \mathrm{E}-01$ & $2.72 \mathrm{E}+02$ & & & \\
\hline 145 & $1.47 \mathrm{E}+00$ & $3.65 \mathrm{E}+02$ & & & \\
\hline 148 & $2.55 \mathrm{E}+00$ & $4.87 E+02$ & & & \\
\hline 150 & $3.65 E+00$ & $5.88 \mathrm{E}+02$ & & & \\
\hline 152 & $5.16 \mathrm{E}+00$ & $7.09 E+02$ & & & \\
\hline 155 & $8.54 \mathrm{E}+00$ & $9.33 \mathrm{E}+02$ & & & \\
\hline
\end{tabular}


M06-2X/cc-pVDZ Optimized Geometries (Cartesians, in $\AA$ ) of Stationary Points

1, СТСТСт-[12]Annulene, C1 symmetry M06-2x/cc-pVDZ geometry

\begin{tabular}{|c|c|c|c|}
\hline \multirow[b]{2}{*}{ Atom } & \multicolumn{3}{|c|}{ Coordinates (Angstroms) } \\
\hline & $\mathrm{X}$ & $\mathrm{Y}$ & Z \\
\hline \multicolumn{4}{|c|}{ 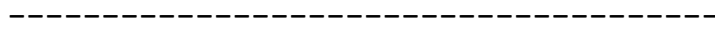 } \\
\hline $\mathrm{C} 1$ & 0.136726 & -1.682414 & 0.276013 \\
\hline $\mathrm{C} 2$ & -1.019951 & -2.123226 & -0.251563 \\
\hline $\mathrm{C} 3$ & -2.336915 & -1.512064 & -0.005489 \\
\hline $\mathrm{C} 4$ & -2.596167 & -0.219074 & 0.274232 \\
\hline $\mathrm{C} 5$ & -1.571920 & 0.841765 & 0.439511 \\
\hline C6 & -1.361078 & 1.893981 & -0.367656 \\
\hline C7 & -0.192718 & 2.776679 & -0.178137 \\
\hline $\mathrm{C} 8$ & 1.066652 & 2.304101 & -0.046803 \\
\hline $\mathrm{C} 9$ & 1.386821 & 0.892187 & -0.241682 \\
\hline C10 & 2.439968 & 0.186204 & 0.218275 \\
\hline C11 & 2.548853 & -1.259885 & -0.020640 \\
\hline C12 & 1.494285 & -2.107617 & -0.060886 \\
\hline H 13 & 0.097196 & -0.869041 & 0.998655 \\
\hline $\mathrm{H} 14$ & -1.002027 & -2.958865 & -0.959575 \\
\hline H 15 & -3.199985 & -2.172078 & -0.133940 \\
\hline $\mathrm{H} 16$ & -3.644697 & 0.074948 & 0.386839 \\
\hline H 17 & -0.901738 & 0.759685 & 1.301610 \\
\hline H 18 & -2.038522 & 2.094110 & -1.205187 \\
\hline H 19 & -0.363388 & 3.856283 & -0.135361 \\
\hline $\mathrm{H} 20$ & 1.879699 & 2.999137 & 0.182062 \\
\hline H2 1 & 0.656849 & 0.349018 & -0.843369 \\
\hline H 22 & 3.243134 & 0.680899 & 0.774532 \\
\hline H23 & 3.549378 & -1.664159 & -0.198677 \\
\hline H 24 & 1.657942 & -3.146208 & -0.362118 \\
\hline
\end{tabular}

2b, СССтСт-[12]Annulene, C1 symmetry M06-2x/cc-pVDz geometry

\begin{tabular}{|c|c|c|c|}
\hline \multirow[b]{2}{*}{ Atom } & \multicolumn{3}{|c|}{ Coordinates (Angstroms) } \\
\hline & $\mathrm{x}$ & $\mathrm{Y}$ & $\mathrm{Z}$ \\
\hline C1 & 0.205143 & -1.718256 & 0.165528 \\
\hline $\mathrm{C} 2$ & -1.050377 & -2.035360 & -0.496201 \\
\hline C3 & -2.150314 & -1.285523 & -0.281514 \\
\hline C4 & -2.218487 & -0.228954 & 0.763358 \\
\hline C5 & -1.861403 & 1.060892 & 0.708970 \\
\hline C6 & -1.484493 & 1.943808 & -0.431898 \\
\hline C7 & -0.292347 & 2.531508 & -0.627354 \\
\hline C8 & 0.959245 & 2.090483 & 0.024459 \\
\hline C9 & 1.358681 & 0.825725 & -0.195270 \\
\hline C10 & 2.513602 & 0.091035 & 0.315305 \\
\hline C11 & 2.590307 & -1.262670 & 0.263914 \\
\hline C12 & 1.455332 & -2.069912 & -0.197710 \\
\hline H13 & 0.121772 & -0.969777 & 0.956425 \\
\hline H 14 & -1.071016 & -2.829834 & -1.246991 \\
\hline H15 & -3.069972 & -1.522286 & -0.826133 \\
\hline H16 & -2.580196 & -0.583684 & 1.737351 \\
\hline H 17 & -1.949001 & 1.610855 & 1.655010 \\
\hline H18 & -2.323950 & 2.275742 & -1.053978 \\
\hline H19 & -0.228730 & 3.340035 & -1.364304 \\
\hline H2O & 627 & 2.790323 & 0.633653 \\
\hline H 21 & 0.718207 & 0.259467 & -0.872530 \\
\hline H 22 & 3.360956 & 0.660955 & 0.706851 \\
\hline H23 & 3.529735 & -1.754959 & 0.529103 \\
\hline H 24 & 1.632271 & -2.868787 & -0.925976 \\
\hline
\end{tabular}

TS for 1-->2b, [12]Annulene, C1 symm M06-2x/cc-pVDz geometry

\begin{tabular}{|c|c|c|c|}
\hline \multirow[b]{2}{*}{ Atom } & \multicolumn{3}{|c|}{ Coordinates (Angstroms) } \\
\hline & $\mathrm{x}$ & $\mathrm{Y}$ & $\mathrm{Z}$ \\
\hline $\mathrm{C} 1$ & 0.120576 & -1.587561 & 0.044006 \\
\hline C2 & -1.113094 & -1.976974 & -0.500553 \\
\hline C3 & -2.317150 & -1.403124 & -0.072636 \\
\hline C4 & -2.390967 & -0.239146 & 0.723972 \\
\hline C5 & -1.581624 & 0.893960 & 0.615123 \\
\hline C6 & -1.255735 & 1.632496 & -0.556888 \\
\hline C7 & -0.221977 & 2.573302 & -0.574297 \\
\hline C8 & 1.002328 & 2.252697 & 0.060772 \\
\hline C9 & 1.371167 & 0.909746 & -0.013840 \\
\hline $\mathrm{C} 10$ & 2.474919 & 0.153168 & 0.412359 \\
\hline C11 & 2.532226 & -1.219663 & 0.137879 \\
\hline $\mathrm{C} 12$ & 1.415035 & -1.983734 & -0.271831 \\
\hline H13 & 0.050844 & -0.856850 & 0.850074 \\
\hline H 14 & -1.147846 & -2.820487 & -1.196708 \\
\hline H15 & -3.228901 & -1.994231 & -0.215805 \\
\hline H16 & -3.096332 & -0.267438 & 1.564184 \\
\hline H 17 & -1.322866 & 1.407825 & 1.550000 \\
\hline $\mathrm{H} 18$ & -1.959076 & 1.564150 & -1.392872 \\
\hline H19 & -0.332652 & 3.512198 & -1.125510 \\
\hline $\mathrm{H} 2 \mathrm{O}$ & 1.682998 & 3.019649 & 0.442090 \\
\hline H 21 & 0.704643 & 0.353796 & -0.665591 \\
\hline H 22 & 3.342515 & 0.642451 & 0.863408 \\
\hline H23 & 3.509639 & -1.708776 & 0.174494 \\
\hline H 24 & 1.582809 & -2.883289 & -0.872161 \\
\hline
\end{tabular}

2a, СтСтСт-[12]Annulene, Cs symmetry M06-2X/cc-pVDZ geometry (reactant for Polyrate calcs on bond shifting)

\begin{tabular}{|c|c|c|c|}
\hline \multirow[b]{2}{*}{ Atom } & \multicolumn{3}{|c|}{ Coordinates (Angstroms) } \\
\hline & $\mathrm{X}$ & $\mathrm{Y}$ & $\mathrm{z}$ \\
\hline $\mathrm{C} 1$ & 0.185733 & 1.750141 & 1.514742 \\
\hline $\mathrm{C} 2$ & 0.185733 & 1.750141 & -1.514742 \\
\hline C3 & -0.377732 & 2.818832 & 0.676699 \\
\hline $\mathrm{C} 4$ & -0.377732 & 2.818832 & -0.676699 \\
\hline C5 & -0.179191 & 0.457718 & -1.460746 \\
\hline $\mathrm{C} 6$ & -0.179191 & 0.457718 & 1.460746 \\
\hline C7 & -0.640173 & -2.428318 & 0.670733 \\
\hline $\mathrm{C} 8$ & -0.640173 & -2.428318 & -0.670733 \\
\hline C9 & 0.590204 & -0.658341 & 1.992764 \\
\hline $\mathrm{C} 10$ & 0.590204 & -0.658341 & -1.992764 \\
\hline $\mathrm{C} 11$ & 0.406392 & -1.930929 & -1.600339 \\
\hline $\mathrm{C} 12$ & 0.406392 & -1.930929 & 1.600339 \\
\hline H 13 & -0.674024 & 3.747543 & 1.175737 \\
\hline $\mathrm{H} 14$ & -0.674024 & 3.747543 & -1.175737 \\
\hline H 15 & 1.034986 & 2.036823 & -2.148146 \\
\hline H16 & 1.034986 & 2.036823 & 2.148146 \\
\hline H 17 & -1.086628 & 0.189510 & 0.924089 \\
\hline H 18 & -1.086628 & 0.189510 & -0.924089 \\
\hline H 19 & -1.486029 & -2.931238 & -1.156518 \\
\hline $\mathrm{H} 20$ & -1.486029 & -2.931238 & 1.156518 \\
\hline H 21 & 1.042477 & -2.704500 & -2.044288 \\
\hline $\mathrm{H} 22$ & 1.042477 & -2.704500 & 2.044288 \\
\hline H23 & 1.401824 & -0.431797 & -2.690464 \\
\hline $\mathrm{H} 24$ & 1.401824 & -0.431797 & 2.690464 \\
\hline
\end{tabular}


TS for 2b-->2a, СССТСТ-[12]Annulene, C1 symmetry M06-2x/cc-pVDz geometry

\begin{tabular}{|c|c|c|c|}
\hline Atom & \multicolumn{2}{|c|}{ 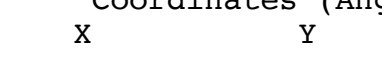 } & $\begin{array}{r}\text { roms ) } \\
\mathrm{Z}\end{array}$ \\
\hline \multicolumn{4}{|c|}{$\begin{array}{l}\text { 3, Bicyclo[ } 6.4 .0] \text { dodeca- } 2,4,6,9,11- \\
\text { pentaene, Cs symmetry } \\
\text { M06-2X/cc-pVDz geometry }\end{array}$} \\
\hline \multicolumn{4}{|c|}{ Coordinates (Angstroms) } \\
\hline Atom & $\mathrm{x}$ & $\mathrm{Y}$ & $\mathrm{Z}$ \\
\hline $\mathrm{C} 1$ & -0.135884 & 1.778055 & 1.419730 \\
\hline 2 & -0.135884 & 1.778055 & -1.419730 \\
\hline 3 & 0.017535 & 2.914256 & 0.733492 \\
\hline 4 & 0.017535 & 2.914256 & -0.733492 \\
\hline 5 & -0.336877 & 0.419468 & -0.788334 \\
\hline 6 & -0.336877 & $0.41946 \varepsilon$ & 0.788334 \\
\hline $\mathrm{C} 7$ & -0.566141 & -2.629847 & 0.672519 \\
\hline $\mathrm{C} 8$ & -0.566141 & -2.629847 & -0.672519 \\
\hline 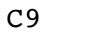 & 0.596456 & -0.569806 & 1.467065 \\
\hline $\mathrm{C} 10$ & 0.596456 & -0.56980 & -1.467065 \\
\hline $\mathrm{C} 11$ & 0.425710 & -1.895756 & -1.480010 \\
\hline C12 & 0.425710 & -1.895756 & 1.480010 \\
\hline H13 & 0.157394 & 3.859403 & 1.260312 \\
\hline H 14 & 0.157394 & 3.859403 & -1.260312 \\
\hline Н15 & -0.114203 & 1.790885 & -2.513244 \\
\hline H16 & -0.114203 & 1.790885 & 2.513244 \\
\hline H 17 & -1.349586 & 0.090685 & 1.077815 \\
\hline Н18 & -1.349586 & 0.090685 & -1.077815 \\
\hline H 19 & -1.261430 & -3.293703 & -1.196846 \\
\hline Н20 & -1.261430 & -3.293703 & 1.196846 \\
\hline H 21 & 1.081881 & -2.504294 & -2.111436 \\
\hline H 22 & 1.081881 & -2.504294 & 2.111436 \\
\hline H23 & 1.414973 & -0.145241 & -2.056629 \\
\hline$n \angle 4$ & 1.414973 & -0.145241 & 2.056629 \\
\hline
\end{tabular}

TS for $2 \mathrm{a}-->3$, Cs symmetry M06-2x/cc-pVDZ geometry

\begin{tabular}{|c|c|c|c|}
\hline \multirow[b]{2}{*}{ Atom } & \multicolumn{3}{|c|}{ Coordinates (Angstroms) } \\
\hline & $\mathrm{X}$ & $\mathrm{Y}$ & $\mathrm{Z}$ \\
\hline C1 & 0.037297 & 1.776343 & 1.480379 \\
\hline $\mathrm{C} 2$ & 0.037297 & 1.776343 & -1.480379 \\
\hline C3 & -0.293826 & 2.878253 & 0.707268 \\
\hline $\mathrm{C} 4$ & -0.293826 & 2.878253 & -0.707268 \\
\hline C5 & -0.180295 & 0.429976 & -1.114214 \\
\hline c6 & -0.180295 & 0.429976 & 1.114214 \\
\hline C7 & -0.677307 & -2.510846 & 0.669006 \\
\hline $\mathrm{C} 8$ & -0.677307 & -2.510846 & -0.669006 \\
\hline C9 & 0.638441 & -0.634938 & 1.689859 \\
\hline $\mathrm{C} 10$ & 0.638441 & -0.634938 & -1.689859 \\
\hline C11 & 0.422484 & -1.946279 & -1.491067 \\
\hline $\mathrm{C} 12$ & 0.422484 & -1.946279 & 1.491067 \\
\hline H13 & -0.235789 & 3.862521 & 1.178832 \\
\hline H 14 & -0.235789 & 3.862521 & -1.178832 \\
\hline H15 & 0.630046 & 1.970260 & -2.382370 \\
\hline н16 & 0.630046 & 1.970260 & 2.382370 \\
\hline H 17 & -1.203114 & 0.142709 & 0.898823 \\
\hline H 18 & -1.203114 & 0.142709 & -0.898823 \\
\hline
\end{tabular}

\begin{tabular}{lrrr} 
H19 & -1.499582 & -3.006035 & -1.198742 \\
H20 & -1.499582 & -3.006035 & 1.198742 \\
H21 & 1.081114 & -2.667929 & -1.985314 \\
H22 & 1.081114 & -2.667929 & 1.985314 \\
H23 & 1.505009 & -0.328505 & -2.284670 \\
H24 & 1.505009 & -0.328505 & 2.284670 \\
\hline
\end{tabular}

4a, Стстстст-[16]Annulene, S4 symmetry M06-2x/cc-pVDz geometry

\begin{tabular}{|c|c|c|c|}
\hline \multirow[b]{2}{*}{ Atom } & \multicolumn{3}{|c|}{ Coordinates (Angstroms) } \\
\hline & $\mathrm{x}$ & $\mathrm{Y}$ & $\mathrm{Z}$ \\
\hline $\mathrm{C} 1$ & 2.333627 & 0.003143 & 0.013509 \\
\hline $\mathrm{C} 2$ & -2.333627 & -0.003143 & 0.013509 \\
\hline C3 & -0.003143 & 2.333627 & -0.013509 \\
\hline C4 & 0.003143 & -2.333627 & -0.013509 \\
\hline C5 & 2.935204 & 1.207287 & -0.536681 \\
\hline C6 & 2.927508 & -1.201672 & -0.114464 \\
\hline C7 & -2.927508 & 1.201672 & -0.114464 \\
\hline $\mathrm{C} 8$ & -2.935204 & -1.207287 & -0.536681 \\
\hline C9 & -1.207287 & 2.935204 & 0.536681 \\
\hline 10 & -1.2016 & -2.92750 & 0.114464 \\
\hline 11 & 1.201672 & 2.927508 & .114464 \\
\hline $\mathrm{C} 12$ & 1.207287 & -2.935204 & 0.536681 \\
\hline $\mathrm{C} 13$ & 2.467648 & 2.470919 & -0.454439 \\
\hline C14 & 2.470919 & -2.467648 & 0.454439 \\
\hline C15 & -2.470919 & 2.467648 & 0.454439 \\
\hline C16 & -2.467648 & -2.470919 & -0.454439 \\
\hline H 17 & 1.418913 & 0.099787 & 0.605014 \\
\hline H18 & -1.418913 & -0.099787 & 0.605014 \\
\hline H19 & -0.099787 & 1.418913 & -0.605014 \\
\hline $\mathrm{H} 20$ & 0.099787 & -1.418913 & -0.605014 \\
\hline H2 1 & 3.905841 & 1.0 & -1.021260 \\
\hline 22 & . & -1 & 45938 \\
\hline 23 & -3.8857 & 1.240119 & -0.645938 \\
\hline H 24 & -3.9058 & -1.062310 & -1.021260 \\
\hline H 25 & -1.062310 & 3.905841 & 1.021260 \\
\hline H2 6 & -1.240119 & -3.885797 & 0.645938 \\
\hline H 27 & 1.240119 & 3.885797 & 0.645938 \\
\hline H28 & 1.062310 & -3.905841 & 1.021260 \\
\hline H29 & 3.121250 & 3.265248 & -0.827372 \\
\hline $\mathrm{H} 30$ & 3.265248 & -3.121250 & 0.827372 \\
\hline H31 & -3.265248 & 3.121250 & 0.827372 \\
\hline & & & \\
\hline
\end{tabular}

4b, СтСтстст-[16]Annulene, C1 symmetry M06-2x/cc-pVDz geometry

\begin{tabular}{|c|c|c|c|}
\hline \multirow[b]{2}{*}{ Atom } & \multicolumn{3}{|c|}{ Coordinates (Angstroms) } \\
\hline & $\mathrm{X}$ & $\mathrm{Y}$ & $\mathrm{Z}$ \\
\hline $\mathrm{C} 1$ & -2.745556 & -1.603352 & -0.037104 \\
\hline $\mathrm{C} 2$ & -2.356579 & -0.309155 & -0.082459 \\
\hline $\mathrm{C} 3$ & -3.120797 & 0.744618 & -0.723795 \\
\hline $\mathrm{C} 4$ & -2.884068 & 2.078904 & -0.651496 \\
\hline $\mathrm{C} 5$ & -1.744119 & 2.737346 & -0.025382 \\
\hline C6 & -0.486036 & 2.244456 & -0.028693 \\
\hline C7 & 0.646091 & 2.830141 & 0.656968 \\
\hline C8 & 1.876317 & 2.257547 & 0.724715 \\
\hline C9 & 2.184850 & 0.985813 & 0.112152 \\
\hline C10 & 3.399138 & 0.407892 & -0.018874 \\
\hline C11 & 3.543159 & -0.826432 & -0.790657 \\
\hline C12 & 2.644009 & -1.831182 & -0.813775 \\
\hline C13 & 1.555214 & -2.038958 & 0.158503 \\
\hline C14 & 0.267248 & -2.303197 & -0.134499 \\
\hline
\end{tabular}




$\begin{array}{lr}\text { C15 } & -0.714126 \\ \text { C16 } & -2.048945 \\ \text { H17 } & -3.683118 \\ \text { H18 } & -1.475438 \\ \text { H19 } & -4.031972 \\ \text { H20 } & -3.652666 \\ \text { H21 } & -1.935957 \\ \text { H22 } & -0.314135 \\ \text { H23 } & 0.479102 \\ \text { H24 } & 2.676899 \\ \text { H25 } & 1.354927 \\ \text { H26 } & 4.298435 \\ \text { H27 } & 4.406186 \\ \text { H28 } & 2.766453 \\ \text { H29 } & 1.851959 \\ \text { H30 } & -0.077397 \\ \text { H31 } & -0.320238 \\ \text { H32 } & -2.675815\end{array}$

0.848885

0.796119

$-0.521215$

0.499764

$-1.237967$

$-1.061316$

0.459110

$-0.607163$

1.159424

1.257438

$-0.342999$

0.340104

$-1.460767$

$-1.573822$

1.214941

$-1.172209$

1.651340

1.483373

TS for $4 \mathrm{~b}-->5 b$, СТСТCTCT to СТСТTCTT[16]Annulene, C1 symmetry M06-2x/cc-pVDz geometry

\begin{tabular}{|c|c|c|c|}
\hline \multirow[b]{2}{*}{ Atom } & \multicolumn{3}{|c|}{ Coordinates (Angstroms) } \\
\hline & $\mathrm{x}$ & $\mathrm{Y}$ & $\mathrm{Z}$ \\
\hline $\mathrm{C} 1$ & -2.828588 & -1.707364 & -0.024216 \\
\hline C2 & -2.351118 & -0.397446 & -0.169273 \\
\hline C3 & -3.119221 & 0.666646 & -0.641490 \\
\hline C4 & -2.854236 & 2.042747 & -0.528886 \\
\hline C5 & -1.701499 & 2.713460 & -0.097980 \\
\hline c6 & -0.418198 & 2.179152 & 0.011741 \\
\hline C7 & 0.682139 & 2.828250 & 0.574187 \\
\hline C8 & 1.976863 & 2.283700 & 0.669262 \\
\hline C9 & 2.274275 & 1.010791 & 0.196209 \\
\hline $\mathrm{C} 10$ & 3.510250 & 0.395561 & -0.011004 \\
\hline C11 & 3.597772 & -0.839370 & -0.679050 \\
\hline C12 & 2.515070 & -1.714640 & -0.826029 \\
\hline C13 & 1.495723 & -1.860179 & 0.126743 \\
\hline C14 & 0.175565 & -2.250781 & -0.122522 \\
\hline C15 & -0.733593 & -2.703118 & 0.841353 \\
\hline C16 & -2.123727 & -2.701368 & 0.667874 \\
\hline H17 & -3.875557 & -1.896587 & -0.284307 \\
\hline H18 & -1.383818 & -0.194522 & 0.289401 \\
\hline H19 & -4.104200 & 0.407706 & -1.044086 \\
\hline $\mathrm{H} 2 \mathrm{O}$ & -3.709881 & 2.687690 & -0.747235 \\
\hline H 21 & -1.8234 & 3.772159 & 0.154053 \\
\hline H22 & -0.266452 & 1.189296 & -0.416683 \\
\hline H23 & 0.521193 & 3.833105 & 0.976388 \\
\hline H 24 & 2.765690 & 2.894807 & 1.115428 \\
\hline H25 & 1.427501 & 0.438523 & -0.176344 \\
\hline H 26 & 4.426983 & 0.964482 & 0.170533 \\
\hline H2 7 & 4.523411 & -1.056634 & -1.219427 \\
\hline H2 8 & 2.465814 & -2.329908 & -1.730734 \\
\hline H29 & 1.754369 & -1.587517 & 1.157243 \\
\hline H30 & -0.196859 & -2.176308 & -1.150922 \\
\hline H31 & & $\$ 871$ & 1.755345 \\
\hline H32 & -2.711574 & -3.467033 & 1.182975 \\
\hline
\end{tabular}

5b, СтСттстт-[16]Annulene, C1 symmetry M06-2x/cc-pVDz geometry

\begin{tabular}{|c|c|c|c|}
\hline \multirow[b]{2}{*}{ Atom } & \multicolumn{3}{|c|}{ Coordinates (Angstroms) } \\
\hline & $\mathrm{X}$ & $\mathrm{Y}$ & $\mathrm{Z}$ \\
\hline $\mathrm{C} 1$ & -2.964150 & -1.614539 & -0.099532 \\
\hline C2 & -2.753946 & -0.207125 & 0.226646 \\
\hline C3 & -3.423766 & 0.820158 & -0.330998 \\
\hline C4 & -3.102872 & 2.232793 & -0.123730 \\
\hline C5 & -1.875027 & 2.780940 & 0.040100 \\
\hline C6 & -0.583334 & 2.114897 & -0.017816 \\
\hline C7 & 0.571045 & 2.738782 & 0.310152 \\
\hline C8 & 1.91330 & 2.158613 & 0.305107 \\
\hline C9 & 2.24366 & .936642 & -0.164232 \\
\hline $\mathrm{C} 10$ & 3.5826 & .363567 & -0.101211 \\
\hline C11 & 3.8872 & -0.944932 & -0.276238 \\
\hline $\mathrm{C} 12$ & 2.88068 & -1.99609 & -0.443338 \\
\hline $\mathrm{C} 13$ & 1.74029 & -1.99229 & 0.274826 \\
\hline $\mathrm{C} 14$ & 0.51371 & -2.715253 & -0.017413 \\
\hline C15 & -0.645903 & -2.207801 & 0.447880 \\
\hline C16 & -1.991796 & -2.548107 & 0.002052 \\
\hline H17 & -3.925579 & -1.890291 & -0.541761 \\
\hline H18 & -1.959515 & 0.037745 & 0.933940 \\
\hline H19 & -4.26860 & 0.602170 & -0.994494 \\
\hline 20 & -3.9529 & .92 & -0.137709 \\
\hline H2 1 & -1.8 & 861338 & .211944 \\
\hline H 22 & -0.5619 & 1.072180 & -0.346320 \\
\hline H23 & 0.50 & 3.776032 & 0.654183 \\
\hline H 24 & 2.70004 & 2.771185 & 0.756305 \\
\hline H 25 & 1.468842 & 0.319307 & -0.626774 \\
\hline H26 & 4.402731 & 1.065389 & 0.079688 \\
\hline H 27 & 4.942118 & -1.229627 & -0.320298 \\
\hline H28 & 3.044392 & -2.757156 & -1.213897 \\
\hline H29 & 1.656896 & -1.252791 & 1.078533 \\
\hline H3O & 0.527234 & -3.534618 & -0.742881 \\
\hline H31 & -0.556145 & -1.358063 & 1.131531 \\
\hline 3 & 2 & 73 & -0 \\
\hline
\end{tabular}

5a, Стсттстт-[16]Annulene, C1 symmetry M06-2x/cc-pVDz geometry

\begin{tabular}{|c|c|c|c|}
\hline \multirow[b]{2}{*}{ Atom } & \multicolumn{3}{|c|}{ Coordinates (Angstroms) } \\
\hline & $\mathrm{X}$ & $\mathrm{Y}$ & $\mathrm{Z}$ \\
\hline $\mathrm{C} 1$ & 0.463780 & -2.430707 & 0.001154 \\
\hline $\mathrm{C} 2$ & -0.452228 & 2.169864 & 0.001838 \\
\hline C3 & 1.605726 & -1.579538 & -0.284997 \\
\hline C4 & -0.777857 & -2.078696 & -0.395871 \\
\hline C5 & 2.828530 & -1.671287 & 0.279174 \\
\hline C6 & -2.014846 & -2.762989 & -0.042047 \\
\hline C7 & 3.876066 & -0.660588 & 0.108697 \\
\hline C8 & -3.234570 & -2.180293 & 0.024068 \\
\hline C9 & 3.661593 & 0.675119 & 0.064712 \\
\hline $\mathrm{C} 10$ & -3.521550 & -0.752653 & -0.131471 \\
\hline C11 & 2.364141 & .299960 & .277650 \\
\hline $\mathrm{C} 12$ & -2.663495 & 226145 & 0.224544 \\
\hline $\mathrm{C} 13$ & 1.987072 & 2.523847 & -0.145384 \\
\hline $\mathrm{C} 14$ & -2.869294 & 1.658712 & 0.051146 \\
\hline C15 & 0.606468 & 3.006076 & -0.056447 \\
\hline C16 & -1.852740 & 2.552645 & 0.010173 \\
\hline H17 & 0.614138 & -3.306262 & 0.641564 \\
\hline H 18 & -0.243626 & 1.097696 & -0.034977 \\
\hline H19 & 0.436375 & 4.086365 & -0.100083 \\
\hline $\mathrm{H} 2 \mathrm{O}$ & -2.082438 & 3.617650 & -0.082754 \\
\hline 121 & 2.716603 & 3.175217 & -0.636930 \\
\hline
\end{tabular}




$\begin{array}{lrrr}\text { H22 } & -3.897161 & 2.013285 & -0.064399 \\ \text { H23 } & 1.628622 & 0.691612 & 0.810570 \\ \text { H24 } & -1.731441 & -0.076312 & 0.705618 \\ \text { H25 } & 4.510625 & 1.334634 & -0.138311 \\ \text { H26 } & -4.500987 & -0.482398 & -0.540781 \\ \text { H27 } & 4.905376 & -1.020801 & 0.026413 \\ \text { H28 } & -4.094136 & -2.827108 & 0.220614 \\ \text { H29 } & 3.060575 & -2.536830 & 0.909606 \\ \text { H30 } & -1.941538 & -3.830379 & 0.186292 \\ \text { H31 } & 1.414877 & -0.749974 & -0.975175 \\ \text { H32 } & -0.880547 & -1.173740 & -1.005399 \\ -\end{array}$

TS $4 \mathbf{c}$ to $4 \mathbf{b}$, TS leading to $4 \mathbf{b}$ from $4 \mathbf{c}$, СтстСтСт-[16]Annulene, C1 symmetry M06-2x/cc-pVDz geometry

\begin{tabular}{|c|c|c|c|}
\hline \multirow[b]{2}{*}{ Atom } & \multicolumn{3}{|c|}{ Coordinates (Angstroms) } \\
\hline & $\mathrm{x}$ & $\mathrm{Y}$ & $\mathrm{Z}$ \\
\hline $\mathrm{C} 1$ & -2.744656 & -1.592798 & -0.036269 \\
\hline $\mathrm{C} 2$ & -2.366515 & -0.295423 & -0.069534 \\
\hline C3 & -3.137164 & 0.755689 & -0.708759 \\
\hline $\mathrm{C} 4$ & -2.901336 & 2.089969 & -0.643098 \\
\hline C5 & -1.756156 & 2.748595 & -0.025958 \\
\hline C6 & -0.499397 & 2.253619 & -0.039266 \\
\hline $\mathrm{C} 7$ & 0.638668 & 2.836454 & 0.639604 \\
\hline $\mathrm{C} 8$ & 1.865065 & 2.257481 & 0.704123 \\
\hline C9 & 2.169884 & 0.981076 & 0.096580 \\
\hline C10 & 3.385873 & 0.406934 & -0.022109 \\
\hline $\mathrm{C} 11$ & 3.549510 & -0.833858 & -0.781983 \\
\hline $\mathrm{C} 12$ & 2.679946 & -1.861780 & -0.792285 \\
\hline C13 & 1.583746 & -2.095832 & 0.173133 \\
\hline C14 & 0.280820 & -2.261261 & -0.121904 \\
\hline C15 & -0.705987 & -2.760746 & 0.836866 \\
\hline C16 & -2.041766 & -2.580829 & 0.781320 \\
\hline H 17 & -3.678127 & -1.891925 & -0.526572 \\
\hline H18 & -1.489150 & -0.013286 & 0.516646 \\
\hline H19 & -4.049688 & 0.430489 & -1.218160 \\
\hline $\mathrm{H} 20$ & -3.671436 & 2.750333 & -1.052093 \\
\hline H 21 & -1.943051 & 3.712307 & 0.460795 \\
\hline H 22 & -0.332454 & 1.344919 & -0.618606 \\
\hline H23 & 0.478604 & 3.795553 & 1.140251 \\
\hline H 24 & 2.670263 & 2.774909 & 1.232458 \\
\hline Н 25 & 1.341446 & 0.437249 & -0.356696 \\
\hline Н26 & 4.279242 & 0.923938 & 0.342779 \\
\hline H2 7 & 4.413861 & -0.884426 & -1.451677 \\
\hline H28 & 2.838084 & -2.652551 & -1.533614 \\
\hline H29 & 1.892902 & -2.198831 & 1.221629 \\
\hline H30 & -0.073304 & -2.070617 & -1.141771 \\
\hline H3 1 & -0.314424 & -3.416007 & 1.621190 \\
\hline Н32 & -2.667277 & -3.181889 & 1.449626 \\
\hline
\end{tabular}

4c, Стстстст-[16]Annulene, C1 symmetry Conformational minimum between $4 \mathrm{a}$ and $4 \mathrm{~b}$

M06-2x/cc-pVDz geometry

\begin{tabular}{|c|c|c|c|}
\hline \multirow[b]{2}{*}{ Atom } & \multicolumn{3}{|c|}{ Coordinates (Angstroms) } \\
\hline & $\mathrm{X}$ & $\mathrm{Y}$ & $\mathrm{Z}$ \\
\hline C1 & 2704743 & -1406573 & -0.022757 \\
\hline $\mathrm{C} 2$ & -2.423240 & -0.182211 & 0.078743 \\
\hline C3 & -3.269856 & 0.847439 & -0.509647 \\
\hline C4 & -3.055739 & 2.182930 & -0.500754 \\
\hline
\end{tabular}

\begin{tabular}{|c|c|c|c|}
\hline C5 & -1.857703 & 2.855469 & -0.005461 \\
\hline C6 & -0.613995 & 2.354671 & -0.147124 \\
\hline C7 & 0.588558 & 2.934777 & 0.417704 \\
\hline $\mathrm{C} 8$ & 1.790378 & 2.314918 & 0.434606 \\
\hline C9 & 2.050607 & 0.993015 & -0.109562 \\
\hline C10 & 3.258804 & 0.401000 & -0.072077 \\
\hline C11 & 3.600428 & -0.912869 & -0.611244 \\
\hline 12 & 2.940045 & -2.086339 & -0.534837 \\
\hline C13 & 1.704702 & -2.490888 & 0.148461 \\
\hline C14 & 0.433380 & -2.054050 & 0.040865 \\
\hline C15 & -0.640653 & -2.776444 & 0.716851 \\
\hline C16 & -1.972589 & -2.577736 & 0.638622 \\
\hline H 17 & -3.605262 & -1.792588 & -0.574090 \\
\hline H18 & -1.572364 & 0.128601 & 0.691119 \\
\hline 19 & -4.204131 & 0.487699 & -0.952013 \\
\hline $\mathrm{H} 2 \mathrm{O}$ & -3.858380 & 2.824911 & -0.874436 \\
\hline H 21 & -1.991687 & 3.820622 & 0.495269 \\
\hline H 22 & -0.508319 & 1.435499 & -0.726539 \\
\hline 23 & 0.492079 & 3.916647 & 0 . \\
\hline H 24 & 2.637516 & 2.820976 & 0.906017 \\
\hline H25 & 1.235686 & 0.466054 & -0.602425 \\
\hline H2 6 & 4.097202 & 0.966877 & 0.350737 \\
\hline H2 7 & 4.584751 & -0.960254 & -1.088734 \\
\hline H28 & 3.475815 & -2.943416 & -0.960008 \\
\hline Н 29 & 1.846849 & -3.382356 & 0.774371 \\
\hline Н 30 & 0.163274 & -1.235023 & -0.629092 \\
\hline Н31 & -0.307001 & -3.641485 & 1.298826 \\
\hline 32 & -2.605387 & -3.334418 & 1.114095 \\
\hline
\end{tabular}

TS for $4 \mathbf{a}$ to $4 \mathbf{c}$, СТСТСТСТ-[16]Annulene, C1 symmetry M06-2x/cc-pVDz geometry

\begin{tabular}{|c|c|c|c|}
\hline \multirow[b]{2}{*}{ Atom } & \multicolumn{3}{|c|}{ Coordinates (Angstroms) } \\
\hline & $\mathrm{x}$ & $\mathrm{Y}$ & $\mathrm{Z}$ \\
\hline C1 & 1.596053 & 1.509378 & 0.08372 \\
\hline $\mathrm{C} 2$ & 2.924874 & 1.276100 & 0.048367 \\
\hline C3 & 3.589894 & 0.200262 & -0.694770 \\
\hline $\mathrm{C} 4$ & 3.282421 & -1.105039 & -0.691491 \\
\hline C5 & 2.296711 & -1.766509 & 0.194063 \\
\hline C6 & 1.124024 & -2.269704 & -0.214953 \\
\hline C7 & 0.188803 & -2.988029 & 0.661432 \\
\hline $\mathrm{C} 8$ & -1.14606 & -2.795779 & 0.655355 \\
\hline C9 & -1.783580 & -1.733286 & -0.097327 \\
\hline C10 & -3.0843 & -1.393372 & 0.005757 \\
\hline C11 & -3.65651 & -0.187795 & -0.582750 \\
\hline $\mathrm{C} 12$ & -3.06639 & 1.030161 & -0.619489 \\
\hline $\mathrm{C} 13$ & -1.811248 & 1.425207 & 0.001680 \\
\hline C14 & -1.358675 & 2.695622 & -0.059353 \\
\hline C15 & -0.173243 & 3.241562 & 0.595648 \\
\hline C16 & 1.068748 & 2.718117 & 0.698514 \\
\hline H 17 & 0.91392 & 0.833587 & -0.438669 \\
\hline $\mathrm{H} 18$ & 3.58485 & 2.016831 & 0.515828 \\
\hline H19 & 4.42 & 758 & -1.322477 \\
\hline 26 & 3.8486 & -1.76 & -1.36 \\
\hline H2 1 & 2.5747 & -1.865910 & 1.250996 \\
\hline H22 & 0.827299 & -2.122364 & -1.259280 \\
\hline H23 & 0.618238 & -3.697888 & 1.373816 \\
\hline H 24 & -1.777579 & -3.384817 & 1.326069 \\
\hline H25 & -1.140359 & -1.114672 & -0.729530 \\
\hline H2 6 & -3.760099 & -2.043153 & 0.573512 \\
\hline H2 7 & -4.671851 & -0.267445 & -0.981530 \\
\hline H2 8 & -3.616365 & 1.840555 & -1.108699 \\
\hline H29 & 1.26683 & 0.691655 & 0.601607 \\
\hline $\mathrm{H} 30$ & -1.9 & 3.428058 & -0.596907 \\
\hline H3 1 & -0.308441 & 4.245118 & 1.011879 \\
\hline
\end{tabular}




\begin{tabular}{|c|c|c|c|}
\hline H32 & 1.812036 & 3.321090 & 1.230095 \\
\hline \multicolumn{4}{|c|}{$\begin{array}{l}\text { TS for } \mathbf{5 b} \text { to } \mathbf{5 a ,} \text {, ССТTCTT-[16]Annulene, } \\
\text { C1 symmetry } \\
\text { M06-2x/cC-pVDz geometry }\end{array}$} \\
\hline \multicolumn{4}{|c|}{ Coordinates (Angstroms) } \\
\hline Atom & $\mathrm{x}$ & $\mathrm{Y}$ & Z \\
\hline 1 & 0.583930 & -2.532750 & 0.012524 \\
\hline 2 & -0.604195 & 2.057255 & -0.023804 \\
\hline 3 & 1.731987 & -1.685533 & -0.243893 \\
\hline $\mathrm{C} 4$ & -0.637473 & -2.094325 & -0.358413 \\
\hline C5 & 2.950939 & -1.739668 & 0.334283 \\
\hline 6 & -1.926256 & -2.707594 & -0.076174 \\
\hline C & 3.933507 & -0.671574 & 0.166206 \\
\hline $\mathrm{C} 8$ & -3.070735 & -2.002684 & -0.133971 \\
\hline C9 & 3.621697 & 0.642286 & 0.043413 \\
\hline 10 & -3.096349 & -0.539996 & -0.407969 \\
\hline C11 & 2.284632 & 1.195576 & 0.196277 \\
\hline $\mathrm{C} 12$ & -3.073292 & 0.377553 & 0.565900 \\
\hline $\mathrm{C} 13$ & 1.856884 & 2.369242 & -0.314387 \\
\hline C14 & -3.057212 & 1.844882 & 0.354831 \\
\hline
\end{tabular}

\begin{tabular}{|c|c|c|c|}
\hline C15 & 0.476408 & 2.840966 & -0.233055 \\
\hline $\mathrm{C} 16$ & -1.958783 & 2.576140 & 0.087197 \\
\hline H 17 & 0.706726 & -3.455042 & 0.589167 \\
\hline H18 & -0.478880 & 0.974666 & 0.078946 \\
\hline H 19 & 0.313318 & 3.912990 & -0.386168 \\
\hline H 20 & -2.073061 & 3.658943 & -0.024739 \\
\hline H 21 & 2.565951 & 3.005459 & -0.853611 \\
\hline H 22 & -4.009271 & 2.370650 & 0.480947 \\
\hline H23 & 1.573990 & 0.591151 & 0.767398 \\
\hline H 24 & -3.085094 & 0.028606 & 1.606700 \\
\hline H25 & 4.428362 & 1.349551 & -0.172247 \\
\hline H 26 & -3.117051 & -0.204688 & -1.452502 \\
\hline H2 7 & 4.988939 & -0.956142 & 0.143620 \\
\hline H28 & -4.022416 & -2.506005 & 0.061128 \\
\hline H 29 & 3.218376 & -2.593909 & 0.965092 \\
\hline Н30 & -1.950820 & -3.765108 & 0.201012 \\
\hline H 31 & 1.538199 & -0.859414 & -0.935168 \\
\hline H32 & -0.679531 & -1.137662 & -0.890194 \\
\hline
\end{tabular}

Complete citation for Gaussian 09:

Gaussian 09, Revision D.01, M. J. Frisch, G. W. Trucks, H. B. Schlegel, G. E. Scuseria, M. A. Robb, J. R. Cheeseman, G. Scalmani, V. Barone, B. Mennucci, G. A. Petersson, H. Nakatsuji, M. Caricato, X. Li, H. P. Hratchian, A. F. Izmaylov, J. Bloino, G. Zheng, J. L. Sonnenberg, M. Hada, M. Ehara, K. Toyota, R. Fukuda, J. Hasegawa, M. Ishida, T. Nakajima, Y. Honda, O. Kitao, H. Nakai, T. Vreven, J. A. Montgomery, Jr., J. E. Peralta, F. Ogliaro, M. Bearpark, J. J. Heyd, E. Brothers, K. N. Kudin, V. N. Staroverov, R. Kobayashi, J. Normand, K. Raghavachari, A. Rendell, J. C. Burant, S. S. Iyengar, J. Tomasi, M. Cossi, N. Rega, J. M. Millam, M. Klene, J. E. Knox, J. B. Cross, V. Bakken, C. Adamo, J. Jaramillo, R. Gomperts, R. E. Stratmann, O. Yazyev, A. J. Austin, R. Cammi, C. Pomelli, J. W. Ochterski, R. L. Martin, K. Morokuma, V. G. Zakrzewski, G. A. Voth, P. Salvador, J. J. Dannenberg, S. Dapprich, A. D. Daniels, O. Farkas, J. B. Foresman, J. V. Ortiz, J. Cioslowski, D. J. Fox, Gaussian, Inc., Wallingford CT, 2009.

Complete citation for POLYRATE-2010A:

J. Zheng, S. Zhang, B. J. Lynch, J. C. Corchado, Y.-Y. Chuang, P. L. Fast, W.-P. Hu, Y.-P. Liu, G. C. Lynch, K. A. Nguyen, C. F. Jackels, A. F. Ramos, B. A. Ellingson, V. S. Melissas, J. Villà, I. Rossi, E. L. Coitiño, J. Pu, T. V. Albu, R. Steckler, B. C. Garrett, A. D. Isaacson, D. G. Truhlar, POLYRATE-Version 2010A, University of Minnesota, Minneapolis, MN, 2010. 